\title{
ARTICLE OPEN \\ Guiding T lymphopoiesis from pluripotent stem cells by defined transcription factors
}

\author{
Rongqun Guo ${ }^{1,2,3,5,6}$, Fangxiao Hu ${ }^{1,2,5,6}$, Qitong Weng ${ }^{1,2,3,5,6}$, Cui Lv ${ }^{1,2,5,6}$, Hongling Wu $\mathrm{u}^{1,2,5,6}$, Lijuan Liu ${ }^{1,2,4,5,6}$, Zongcheng Li iD ${ }^{7}$, \\ Yang Zeng ${ }^{7}$, Zhijie Bai (D)', Mengyun Z'hang ${ }^{1,2,4,5,6}$, Yuting Liu ${ }^{1,2,5,6}$, Xiaofei Liu ${ }^{1,2,4,5,6}$, Chengxiang Xia ${ }^{1,2,3,5,6}$, Tongjie Wang ${ }^{1,2,5,6^{\prime}}$ \\ Peiqing Zhou ${ }^{1,2,3,5,6}$, Kaitao Wang ${ }^{1,2,4,5,6}$, Yong Dong ${ }^{1,2,5,6}$, Yuxuan Luo ${ }^{8}$, Xiangzhong Zhang ${ }^{8}$, Yuxian Guan ${ }^{1,2,5,6}$, Yang Geng ${ }^{1,2,4,5,6}$,

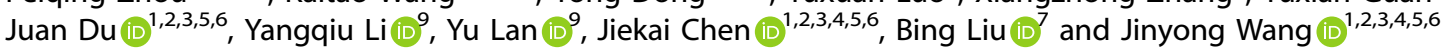

\begin{abstract}
Achievement of immunocompetent and therapeutic T lymphopoiesis from pluripotent stem cells (PSCs) is a central aim in T cell regenerative medicine. To date, preferentially reconstituting T lymphopoiesis in vivo from PSCs remains a practical challenge. Here we documented that synergistic and transient expression of Runx1 and Hoxa9 restricted in the time window of endothelial-tohematopoietic transition and hematopoietic maturation stages in a PSC differentiation scheme (iR9-PSC) in vitro induced preferential generation of engraftable hematopoietic progenitors capable of homing to thymus and developing into mature T cells in primary and secondary immunodeficient recipients. Single-cell transcriptome and functional analyses illustrated the cellular trajectory of T lineage induction from PSCs, unveiling the T-lineage specification determined at as early as hemogenic endothelial cell stage and identifying the bona fide pre-thymic progenitors. The induced T cells distributed normally in central and peripheral lymphoid organs and exhibited abundant TCRaß repertoire. The regenerative $\mathrm{T}$ lymphopoiesis restored immune surveillance in immunodeficient mice. Furthermore, gene-edited iR9-PSCs produced tumor-specific T cells in vivo that effectively eradicated tumor cells. This study provides insight into universal generation of functional and therapeutic T cells from the unlimited and editable PSC source.
\end{abstract}

Cell Research (2020) 30:21-33; https://doi.org/10.1038/s41422-019-0251-7

\section{INTRODUCTION}

A prominent method to generate T cells is an in vitro system via co-culture of either mouse or human hematopoietic stem/ progenitors (HSPCs) with stromal cell lines expressing the Notch ligand, such as OP9-DL1/DL4 or 3D-based MS5-hDLL1/4. ${ }^{1-3}$ Despite the great contribution of this approach to studying $T$ cell development in vitro, phenotypic $T$ cells produced by this approach face severe immunocompetency problems in vivo after engraftment, due to the inadequate in vitro recapitulation of natural thymus microenvironment. Natural mouse $\mathrm{Sca}^{+}{ }^{+} \mathrm{CKit}^{+}$and human $\mathrm{CD}_{3} 4^{+}$blood progenitor cells can be induced into $\mathrm{CD} 7^{+}$ pre-thymic cells in vitro, which successfully colonize thymi and mature into immunocompetent T cells in vivo. ${ }^{4,5}$ However, this in vitro plus in vivo two-step approach never succeeded in achieving induced $\mathrm{T}$ lymphopoiesis when starting from pluripotent stem cells (PSCs), as induced T cell progenitors from PSCs showed intrinsic thymus-homing defect in vivo. ${ }^{6}$ Another prevailing concept for generating functional $T$ lymphopoiesis from PSCs is via induction of hematopoietic stem cell (HSC)-like intermediates followed by in vivo multi-lineage hematopoiesis, including $\mathrm{T}$ cells. ${ }^{7-10}$ However, generating robust bona fide induced-HSC (iHSC) from PSCs remains inefficient ${ }^{11,12}$ and whether this approach can generate therapeutic tumor-killing T cells is unknown. Recently, Hoxb5 is shown to convert natural B cells into functional T cells in vivo, ${ }^{13}$ providing an alternative method to shorten the immune system recovery gap in conventional HSC transplantation. Nonetheless, a solid and universal approach, capable of generating immunocompetent and therapeutic $\mathrm{T}$ lymphopoiesis from the unlimited and geneeditable PSCs, is still lacking.

Accumulated developmental evidence shows that blood progenitors prior to the occurrence of definitive HSCs, also possess $T$ cell lineage differentiation potential. ${ }^{14-17}$ Despite the abundant knowledge of the pivotal transcription factors regulating T cell development from HSC derivatives, ${ }^{18}$ intrinsic determinants of $T$ cell lineage potential in the HSC-independent hematopoietic progenitors at the pre-liver and pre-thymus stages remain elusive. Thus, identifying such crucial $T$ lineage-potential

\footnotetext{
${ }^{1}$ State Key Laboratory of Experimental Hematology, CAS Key Laboratory of Regenerative Biology, Guangzhou Institutes of Biomedicine and Health, Chinese Academy of Sciences, Guangzhou, China; ${ }^{2}$ Guangzhou Regenerative Medicine and Health-Guangdong Laboratory (GRMH-GDL), Guangzhou, China; ${ }^{3}$ University of Chinese Academy of Sciences, Beijing, China; ${ }^{4}$ Joint School of Life Sciences, Guangzhou Institutes of Biomedicine and Health, Guangzhou Medical University, Guangzhou, China; ${ }^{5}$ Guangdong Provincial Key Laboratory of Stem cell and Regenerative Medicine, Guangzhou Institutes of Biomedicine and Health, Chinese Academy of Sciences, Guangzhou, China; ${ }^{6}$ Institute for Stem Cell and Regeneration, Chinese Academy of Sciences, Beijing, China; ${ }^{7}$ State Key Laboratory of Proteomics, Translational Medicine Center of Stem Cells, Fifth Medical Center, General

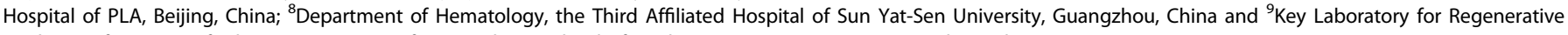
Medicine of Ministry of Education, Institute of Hematology, School of Medicine, Jinan University, Guangzhou, China

Correspondence: Bing Liu (bingliu17@yahoo.com) or Jinyong Wang (wang_jinyong@gibh.ac.cn)

These authors contributed equally: Rongqun Guo, Fangxiao Hu, Qitong Weng
}

Received: 4 July 2019 Accepted: 15 October 2019

Published online: 15 November 2019 
determinants might help to establish a solid protocol for efficiently reconstituting $T$ lymphopoiesis from PSCs.

In this study, by using a unbiased in vivo functional screening approach, we identified that the coordinated and transient expression of exogenous Runx 1 and Hoxa9 at the early time window from endothelial-to-hematopoietic transition stage to hematopoietic progenitor maturation stage in a PSC differentiation scheme in vitro, produced a type of induced hematopoietic progenitor cells (iHPCs) with thymus-homing features, which was engraftable and gave rise to induced $T$ cells (iT cells) with abundant TCRa $\beta$ repertoire in immunodeficient mice. Physiologically, the iT cells successfully restored immune surveillance function in immunodeficient mice. Therapeutically, these iT cells possessed anti-tumor activities in vivo when engineered to carry tumor antigen-specific TCR at PSC stage. For the first time, we establish a novel approach of preferentially generating functional and therapeutic $\mathrm{T}$ lymphopoiesis in vivo from PSCs, which technically creates a link between the unlimited and editable PSC source and T cell-based immunotherapy for translational purpose.

\section{RESULTS}

Reconstitution of T lymphopoiesis in vivo from inducible Runx1p2a-Hoxa9-embryonic stem cells

We hypothesized that the lymphogenic potential is determined by intrinsic determinants at putative endothelial precursor cell stage prior to and independent of HSC formation. Therefore, enforced expression of these master determinants might direct lymphoid differentiation from PSCs. Since Runx1 is pivotal for endothelial to hematopoietic transition (EHT) ${ }^{19-21}$ definitive hematopoietic development $^{22-24}$ and $\mathrm{T}$ cell development, ${ }^{18}$ we started from evaluating the potential effect of Runx 1 in lymphogenic commitment from PSCs. To avoid expression variations introduced by viral delivery systems, we inserted the inducible expression cassette of Runx 1 into the Rosa26 locus of mouse embryonic stem cells (iRunx1-ESC, C57BL/6 background) by homologous recombination (Supplementary information Fig. S1a), which resulted in the conditional expression of exogenous Runx 1 in the presence of doxycycline (Supplementary information Fig. S1b). We used AFT024-(mSCF/mIL3/mlL6/hFlt3L) cell line-cultured supernatants as conditioned medium (CM) for the in vitro induction of induced hemogenic endothelial progenitors (iHECs) and subsequent iHPC, as AFT024 CM is beneficial for the generation of induced HPCs in vitro. ${ }^{25}$ To functionally assess the T lymphopoiesis potential of iHPCs, we transplanted the bulk cells containing abundant iHPCs (referred as iHPC thereafter) into irradiated (2.25 Gy) B-NDG recipients (iHPC recipients) and used the occurrence of $\mathrm{CD}^{+}$ cells in peripheral blood (PB) as a positive readout of induced $T$ lymphopoiesis in vivo (Fig. 1a). Based on a modified protocol for HEC induction from PSCs, ${ }^{26}$ we successfully generated iHECs and hematopoietic progenitor derivatives (Supplementary information Fig. S1c-e). However, the iRunx1-ESC derivatives eventually failed to generate T cells on the conditions of either in vitro OP9-DL1 coculture system (Supplementary information Fig. S1f) or in vivo transplantation setting (Supplementary information Fig. S1g). We speculated that the expression of other transcription factors essential for $\mathrm{T}$ lineage generation might be absent in the iRunx1ESC derivatives. To identify these absent factors, we sorted single iHECs from iRunx1-ESCs and performed single-cell RNA-Seq. In comparison with E11 T1-pre-HSCs $\left(\mathrm{CD} 31^{+} \mathrm{CD} 41^{\text {low }} \mathrm{CD} 45^{-} \mathrm{c}\right.$ $\left.\mathrm{kit}^{+} \mathrm{CD} 201^{\text {high }}\right)$, we identified eight hematopoietic-essential tran-

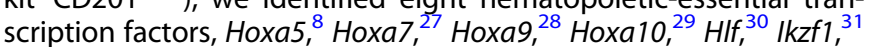
Nkx2-3, ${ }^{32}$ and Setbp 1, ${ }^{33}$ which were barely expressed in iRunx1ESC-derived iHECs but abundantly expressed in E11 T1-pre-HSCs (Fig. 1b), consistent with the previous reports that human PSCderived HECs lack expression of HOXA family. ${ }^{8,34}$ We further used an "iRunx $1+X i$ " tandem-factor-knock-in strategy to perform unbiased screening of the potential combinatory effects of these factors with Runx 1 in lymphoid lineage induction. Following the same induction protocol, we identified that the inducible expression of exogenous Runx 1 and Hoxa9 from day 6 to day 11 during the induction program led to the production of robust iHECs phenotypically resembling embryonic pre-HSCs

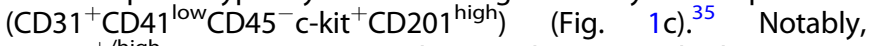
CD201+/high expression can be used to enrich hemogenic precursors with both definitive HPC and HSC potential from as early as E9.5 embryos. ${ }^{36}$ After co-culture of these iHECs with OP9$\mathrm{DL} 1$ feeder line $\left(\mathrm{GFP}^{+}\right)$in the presence of $\mathrm{CM}$ and doxycycline (1 $\mu \mathrm{g} / \mathrm{mL}$ ), robust iHPC occurred at day 21 , including phenotypic prethymic progenitors ( Lin $\left.^{-} \mathrm{c}-\mathrm{kit}^{+} \mathrm{CD} 127^{+} / \mathrm{CD} 135^{+}\right)^{18}$ (Fig. 1d), and $\mathrm{CD} 1 \mathrm{~b}^{+} / \mathrm{Gr}^{+}$myeloid cells, but no $\mathrm{CD}^{+} \mathrm{T}$ cells (Supplementary information Fig. S1h). To further assess the engraftment potential of these iHPCs, we transplanted 0.5-1 million iR9-ESC-derived iHPCs (day-21) into irradiated (2.25 Gy) B-NDG mice (8-week-old, CD45.1 strain) in the absence of doxycycline. Four weeks after transplantation, we observed donor-derived CD45.2 $2^{+} \mathrm{CD}^{+} \mathrm{T}$ cells, but no $\mathrm{CD} 45.2^{+} \mathrm{CD}_{19}{ }^{+} \mathrm{B}$ cells and no $\mathrm{CD} 45.2^{+} \mathrm{CD} 11 \mathrm{~b}^{+}$myeloid cells, in the PB of B-NDG mice transplanted with the iHPCs (Fig. 1e). Five independent experiments indicated that the iR9ESC-derived iHPCs gave rise to $\mathrm{CD}^{+}$iT cells in over $80 \%$ B-NDG recipients (iT-B-NDG mice, 32/40) (Fig. 1f; Supplementary information Fig. S1i). In addition, the day-17 iHPC also reconstituted T lymphopoiesis in B-NDG recipients (Supplementary information Fig. S2a-d). Thus, we established a novel approach of preferentially generating iT cells from gene-edited PSCs by defined transcription factor Runx1 and Hoxa9.

The iT cells show features of multi-organ distributions and abundant TCR diversity

We further analyzed the tissue distributions and immunophenotypes of the regenerated T lymphocytes in iT-B-NDG mice. Mature CD4SP and CD8SP iT cells were detected in the spleen, lymph node and PB of iT-B-NDG mice, the majority of which were TCR $\beta$ positive (Fig. 2a). In addition, $\gamma \delta$ iT cells were also detected in gut and lung tissues of iT-B-NDG mice (Supplementary information Fig. S3a). Induced NK cells (iNK, CD45.2 ${ }^{+} \mathrm{NK} 1.1^{+} \mathrm{CD}^{-}$) were also detected in the spleen and bone marrow of iT-B-NDG mice (Supplementary information Fig. S3b). The thymus of iT-B-NDG mice also contained induced CD4SP (iCD4SP), induced double positive (iDP, CD45.2 $2^{+} \mathrm{CD} 4{ }^{+} \mathrm{CD} 8^{+}$), induced CD8SP (iCD8SP), and induced double negative (iDN, $\mathrm{CD} 45.2^{+} \mathrm{Lin}^{-} \mathrm{CD} 4^{-} \mathrm{CD} 8^{-}$) cells when examined at week 4 and week 5 after transplantation of iHPC. Interestingly, the majority of the iDN cells were at iDN1 $\left(\mathrm{CD} 45.2^{+} \mathrm{Lin}^{-} \mathrm{CD}^{-} \mathrm{CD}^{-} \mathrm{CD}^{+} 4^{+} \mathrm{CD} 25^{-}\right)$phase at week 4 , and at iDN2 (CD45.2 $\left.{ }^{+} \mathrm{Lin}^{-} \mathrm{CD} 4{ }^{-} \mathrm{CD} 8^{-} \mathrm{CD}_{4}{ }^{+} \mathrm{CD} 25^{+}\right) / \mathrm{iDN} 3\left(\mathrm{CD} 45.2^{+} \mathrm{Lin}^{-}\right.$ $\mathrm{CD} 4^{-} \mathrm{CD} 8^{-} \mathrm{CD}_{4}{ }^{-} \mathrm{CD} 25^{+}$) phases at week 5 (Fig. 2b). Besides the iT cells and induced NK1.1 ${ }^{+} \mathrm{CD}^{-} \mathrm{NK}$ (iNK) cells detected in bone marrow, we also observed iR9-ESC-derived $\mathrm{Lin}^{-} \mathrm{Sca}^{+}{ }^{+} \mathrm{CKit}^{+}$(iLSK) progenitor cells (Fig. 2c). To assess whether the iLSK cells can contribute to $\mathrm{T}$ lymphopoiesis, we sorted this population from primary iHPC recipients (week 6) and performed secondary transplantation. Six weeks after transplantation, iT cells appeared in $\mathrm{PB}, \mathrm{BM}$, and SP of the B-NDG recipients (Fig. 2d). Of note, despite iR9-ESC-derived myeloid lineage cells were barely detected in vivo, the iLSK cells indeed gave rise to very limited myeloid colonies in CFU assay (data not shown). To further characterize the iT cells at transcriptome level, we sorted 1000 cell aliquots of the CD4SP iT cells and CD8SP iT cells from the spleens of iT-B-NDG mice for RNA-Seq analysis. Our data indicated that the CD4SP iT cells resembled natural CD4SP T cells, and the CD8SP iT cells resembled natural CD8SP T cells, both of which expressed surface marker-encoding genes $C d 2$, Fas, Cd3e, Cxcr3, Cd28, Cd27, $C d 7, C d 5$, and II7r (Fig. 2e). Of note, the CD4SP iT cells, but not CD8SP iT cells, expressed the ThPOK (T-helper-inducing POK factor, also known as Zbtb7b), a master regulator in regulating CD4 vs 
a

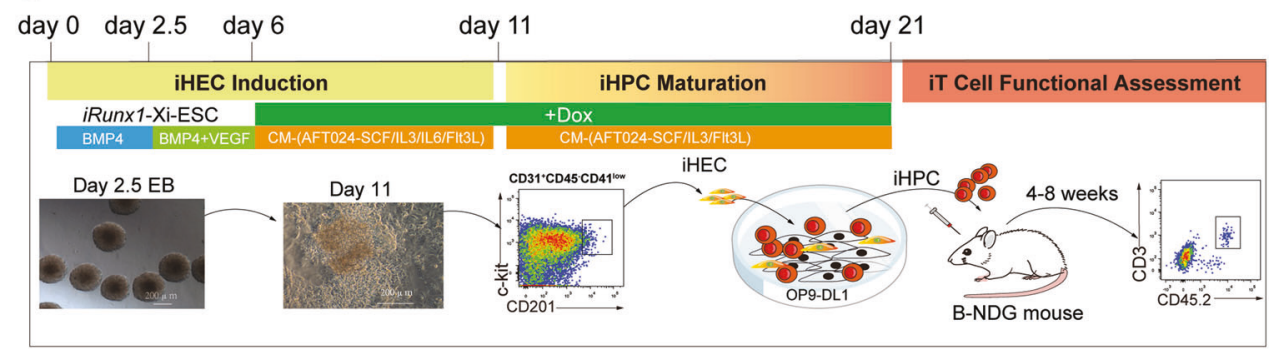

b

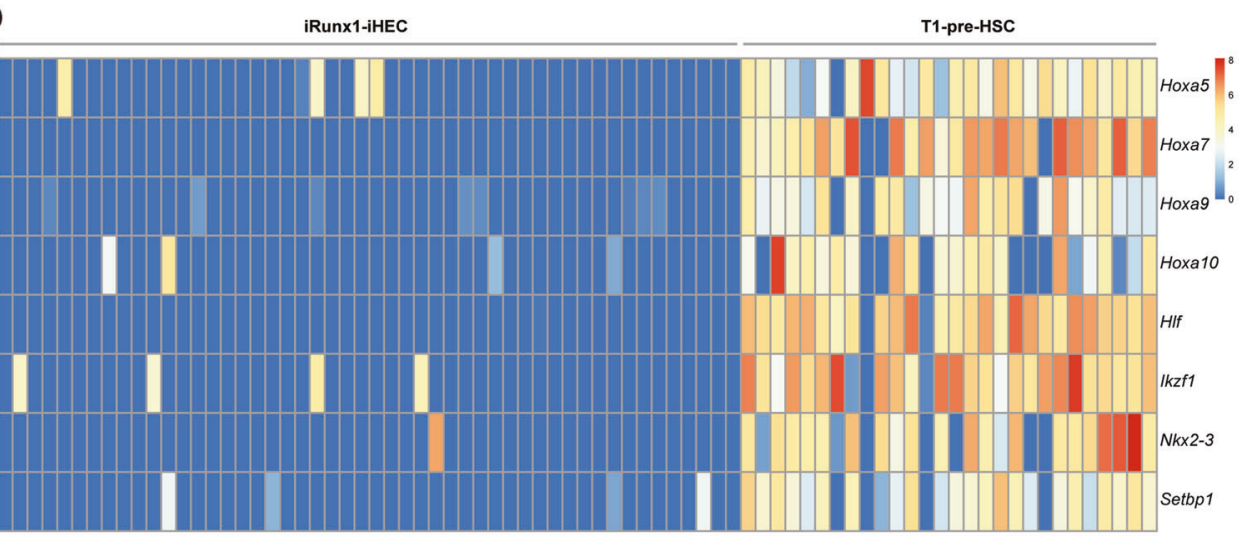

C

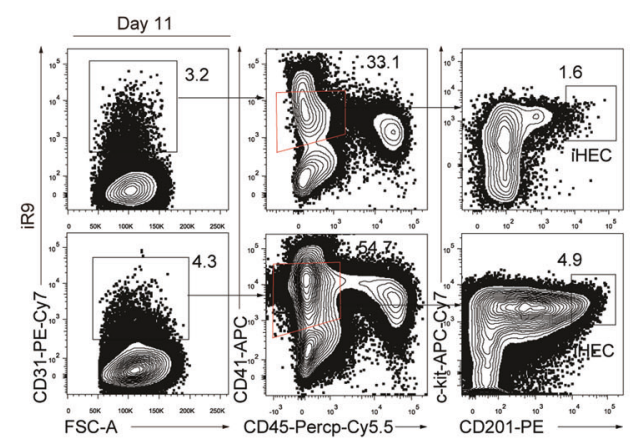

d

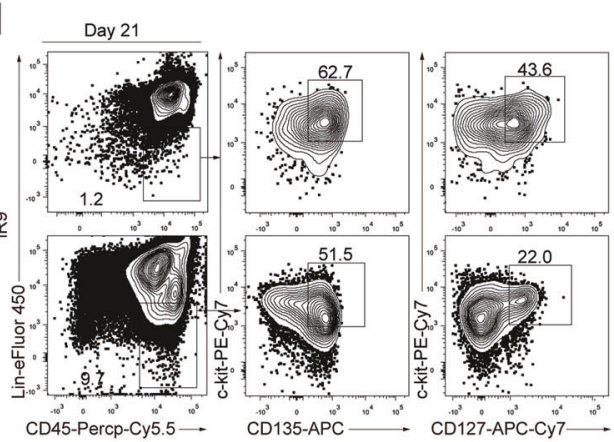

e
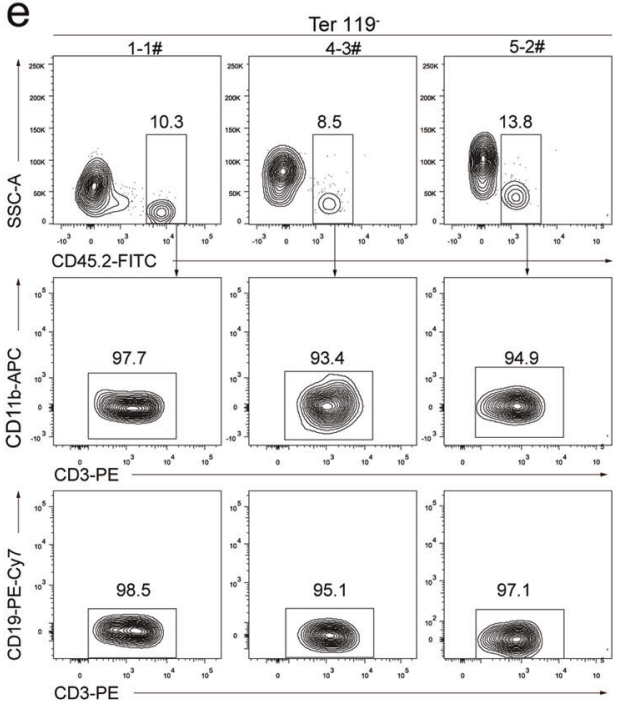

$f$

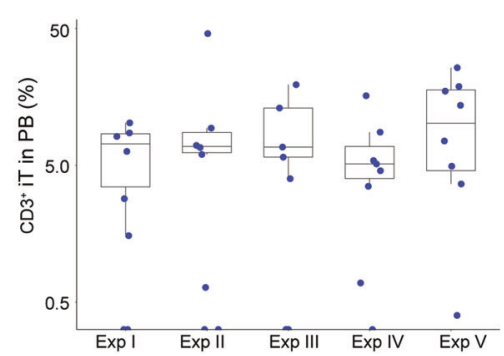

two-fold; adjusted $\mathrm{P}$ value $<0.05$ (DESeq2 R package)) (Supplementary information Table S1), including weaker expression of Tcf7. Genomic PCR sequencing using primer pairs flanking the Runx1-p2a-Hoxa9 element further confirmed that the reconstituted iT cells in vivo were of iR9-PSC origin, which carried the 


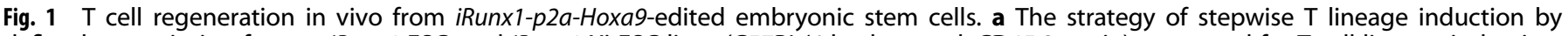

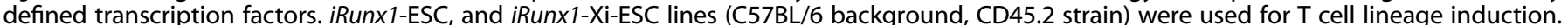

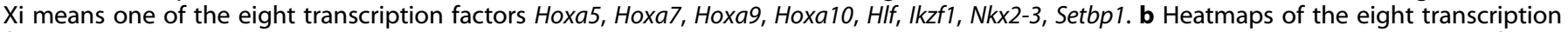

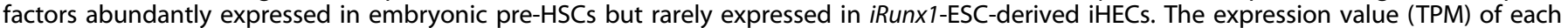

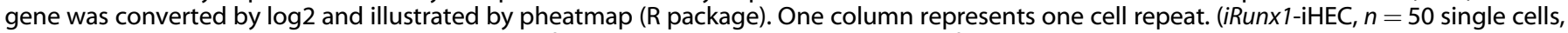

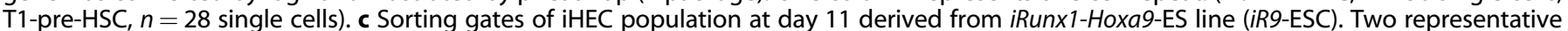

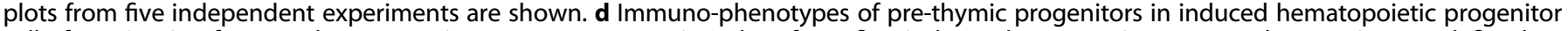

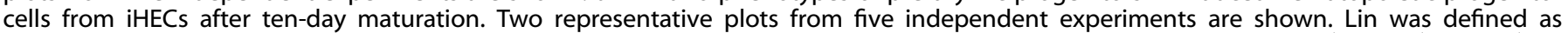
$\mathrm{CD} 2^{-} \mathrm{CD} 3^{-} \mathrm{CD} 4^{-} \mathrm{CD} 8^{-} \mathrm{CD} 11 \mathrm{~b}^{-} \mathrm{Gr}^{-} 1^{-}$Ter119 $^{-} \mathrm{CD} 19^{-} \mathrm{NK} 1.1^{-} \mathrm{TCR} \gamma \delta^{-}$. Pre-thymic progenitors were defined as $\mathrm{Lin}^{-} \mathrm{c}-\mathrm{kit}^{+} \mathrm{CD} 127^{+} / \mathrm{CD} 135^{+}$. e Pluripotent stem cell-derived T cells in peripheral blood (PB) of B-NDG mice were analyzed by flow cytometry 4 weeks after transplantation. One million iHEC-derived hematopoietic cells were transplanted into individual B-NDG mice (CD45.1 $\left.{ }^{+}\right)$irradiated by X-ray $(2.25$ Gy). Three representative mice from five independent experiments were analyzed. $\mathbf{f}$ Summary of pluripotent stem cell-derived T cells in PB of individual B-NDG mice from five independent experiments. Forty B-NDG mice transplanted with ESC-derived iHPCs were analyzed. The box plot shows the percentage of the $\mathrm{CD}^{+}$iT cells in $\mathrm{PB}$, the percentage values were illustrated by ggplot2 (R package). A base-10 logarithmic scale was used for the Y-axis. One point represents one mouse

inserted Runx1-p2a-Hoxa9 element (Supplementary information Fig. S3c). To further assess the diversities of the TCRaß clonotypes of the iT cells, we performed TCR deep sequencing using the sorted naïve CD4SP $\left(\mathrm{CD} 45.2^{+} \mathrm{CD}^{+}{ }^{+} \mathrm{CD}_{\left.22 \mathrm{~L}^{+} \mathrm{CD} 44^{-}\right) \text {and } \mathrm{CD} 8 \mathrm{SP}}\right.$ iT cells (CD45.2 $\left.{ }^{+} \mathrm{CD} 8^{+} \mathrm{CD}_{2} \mathrm{~L}^{+} \mathrm{CD} 44^{-}\right)$from the spleens and thymi of iT-B-NDG mice at week 6 after transplantation of iHPCs. The aliquots of 15,000 sorted naïve CD4SP and CD8SP iT cells were used as cell inputs for TCRa $\beta$ sequencing at transcription level. TCRa $\beta$ clonotype profiling using $\mathrm{MiXCR}^{45}$ captured abundant diversities of TCRa $\beta$ sequences among the sorted naïve iT cells isolated from the thymi (Fig. $2 \mathrm{~g}, \mathrm{~h}$ ) and spleens (Fig. 2i, j) of the iTB-NDG mice. Collectively, these data indicate that the iR9-ESCderived iHPCs reconstitute $\mathrm{T}$ lymphopoiesis in vivo resembling natural $T$ cell development.

Single iHECs efficiently give rise to iT cells both in vitro and in vivo To further investigate the differentiation efficiency of iHECs into iT cells, we sorted single iHECs into individual wells ( 24 well-plates) pre-seeded with OP9-DL1 feeder cells (Fig. 3a). After ten-day coculture, over $15 \%$ individual iHECs formed blood colonies (76/384 wells) (Fig. 3b), which contained abundant pre-thymic progenitors ( Lin $^{-}$c-kit ${ }^{+} \mathrm{CD} 127^{+} / \mathrm{CD}_{135^{+}}$) (Supplementary information Fig. S4). After co-culture with OP9-DL1 feeder line in the presence of hFlt3L and hIL7, these iHEC-formed blood colonies (30/30) further differentiated into $\mathrm{CD}^{+}{ }^{+} \mathrm{iT}$ cells in vitro (Fig. 3b), including a major population of TCRy $\delta$ iT cells, and a small proportion of CD8 ${ }^{+}$ TCR $\beta$ iT cells (Fig. $3 c$ ). To assess the T lymphopoiesis potential of these single-iHEC-derived iHPCs, we further collected the iHPCs from each colony at day 21 and transplanted them into individual B-NDG mice. Four weeks after transplantation, $\mathrm{CD}_{11 \mathrm{~b}^{-} \mathrm{CD}^{-} 9^{-} \mathrm{CD}^{+}} \mathrm{iT}^{\mathrm{iT}}$ cells were detected in approximately $28 \%(7 / 25)$ B-NDG mice transplanted with the cell derivatives from individual iHEC-formed clones (Fig. 3b, d). Collectively, the iR9-ESC-derived iHECs robustly gave rise to $T$ cells at single cell level.

Cellular trajectory from iHECs to pre-thymic progenitors

To characterize the single iHECs at transcriptome level, we performed single-cell RNA-Seq using the sorted iHECs and compared them with natural single E11 endothelial cells (ECs), Type-I pre-HSCs, Type-II pre-HSCs, E12 HSCs, E14 HSCs, and adult HSCs described previously. ${ }^{35}$ Principle component analysis indicated that the iHECs localized between embryonic ECs and preHSCs (Fig. 4a, b). A large proportion of iHECs expressed artery or vein-related genes, suggestive of their EC-like nature (Fig. 4c). Most iHECs expressed endothelial surface marker-encoding genes Cdh5 (coding VE-Cadherin, 70/70) and Esam (57/70), which were continuously expressed from embryonic EC to pre-HSC at a relatively high level. On the other hand, partial iHECs expressed Procr (coding CD201, 32/70), Cd47 (33/70) and Cd63 (44/70), which were upregulated from EC to pre-HSC (Fig. 4d). The expression of transcription factors related to endothelial and hematopoietic development further revealed that the iHECs shared a similar feature with embryonic ECs and pre-HSCs. The majority of the iHECs expressed Fli1 (66/70), Erg (42/70), Lmo2 (49/70), Mycn (65/ $70)$, and Sox7 (38/70). Specifically, a small proportion of iHECs expressed Bcl11a (11/70) and Hoxb5 (24/70). All these transcription factors are pivotal for lymphoid lineage development (Fig. 4e). Thus, the molecular features of the iHECs show similarities with embryonic ECs and pre-HSCs.

To further characterize the iHPC during the hematopoietic maturation process, we sorted the single iHPCs from day-14, day17 , day-21 cell products derived from iR9-ESCs and performed single-cell RNA-Seq. To visualize the time course data of iHPCs, we performed $t$-distributed stochastic neighbor embedding ( $t-S N E$, the genes with expression value TPM $>1$ in more than 30 samples were selected) analysis and illustrated that the day-14 iHPCs formed a unique population distinct from day-11 iHECs and the major population of day-17 iHPCs. However, the day-17 iHPCs and day-21 iHPCs merged already (Fig. 4f). In addition, part of the day21 iHPCs formed a new subpopulation labeled with relatively abundant Gata2 expression (Supplementary information Fig. S5a), indicating the heterogeneity of the iHPCs. The endothelia-related transcription factors, such as Sox7 and Sox18, were abundantly expressed in day-11 iHECs, however, were immediately silenced in day-14 iHPCs (Fig. 4g). The Ets1 gene, involved in embryonic endothelial and lymphoid development, ${ }^{46}$ was shut down in day14 iHPCs but turned on again in day-17 iHPCs (Fig. 4g). The transcription factors involving hematopoietic development, such as Lyl1, ${ }^{47}$ Etv6 $^{48}{ }^{48}$ Prdm5, ${ }^{9}$ Myb $^{49}{ }^{4 f p i 1},{ }^{50-52}$ and Meis1, ${ }^{53}$ were widely expressed among day-14, -17 , and -21 iHPC populations. (Fig. 4h). Further, the transcription factors related to lymphoid development, including $\mathrm{Lmo}^{5}{ }^{54} \mathrm{BCl}_{11} \mathrm{a}^{55} \mathrm{lkzf1}^{56}{ }^{5 \mathrm{Mc}}{ }^{18,57} \mathrm{Gata}^{3}{ }^{58}$ and $T c f 7,{ }^{38}$ were also expressed in iHPCs (Fig. 4i; Supplementary information Fig. S5b). Of note, day-17 and day-21 iHPCs showed abundant expression of Tcf7 (Fig. 4i). Given the thymus-homing problem of the PSC-derived HPCs reported by others, ${ }^{6}$ we observed that the day-21 iHPCs derived from iR9-ESCs abundantly expressed surface marker-encoding genes $\mathrm{Kit}^{18}{ }^{18} \mathrm{Flt} 3{ }^{18}{ }^{18} \mathrm{Cd} 7,{ }^{4,5}$ $\mathrm{Ccr} 9,{ }^{59,60}$ and $\mathrm{CxCr} 4{ }^{61,62}$ which is a feature of natural pre-thymic progenitors possessing thymus-homing ability (Fig. 4j). However, the subpopulation with relatively abundant Gata2 expression at day 21 lacks the thymus-homing feature genes, indicating that these cells unlikely contributed to the regenerated iT lymphopoiesis. Collectively, the iR9-ESC-derived iHPCs show hematopoietic or lymphopoietic features at transcriptome level and the day-21 iHPC population contains robust pre-thymic progenitor-like cells.

The iT cells reject allogeneic skin and form memory response in vivo

To investigate the function of iT cells derived from iR9-ESC (C57BL/ 6 background) in vivo, we transferred the iT cells (equivalent to 5 million iT cells per $\operatorname{Rag}^{-1-}$ mouse) isolated from iT-B-NDG spleen into $\operatorname{Rag} 1^{-1-}$ mice (iT-Rag $1^{-1-}$ mice). Four days after the adoptive 


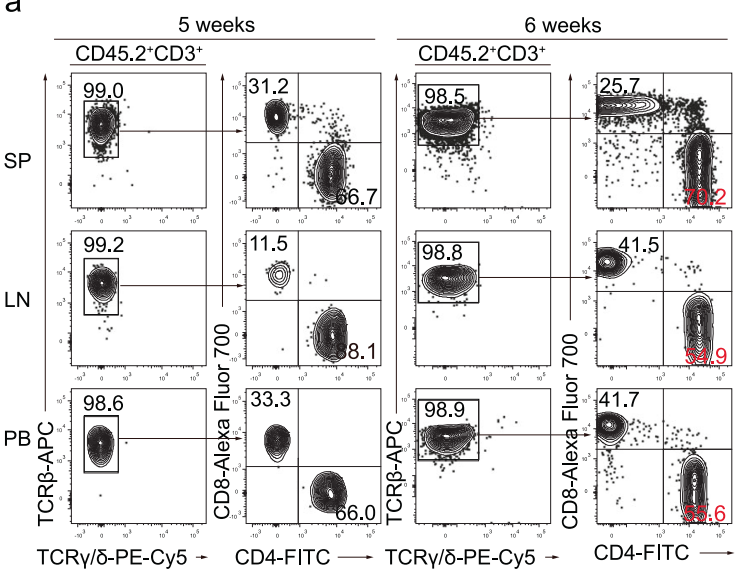

C

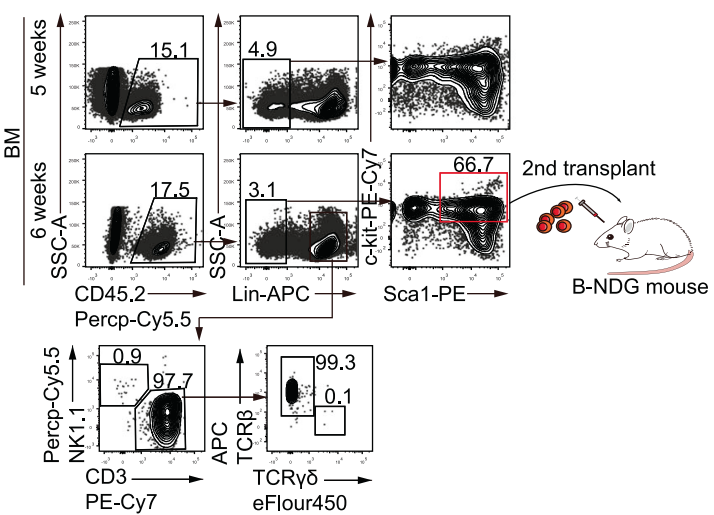

e

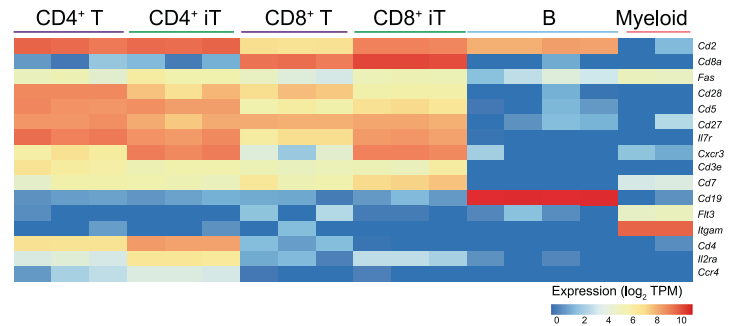

f

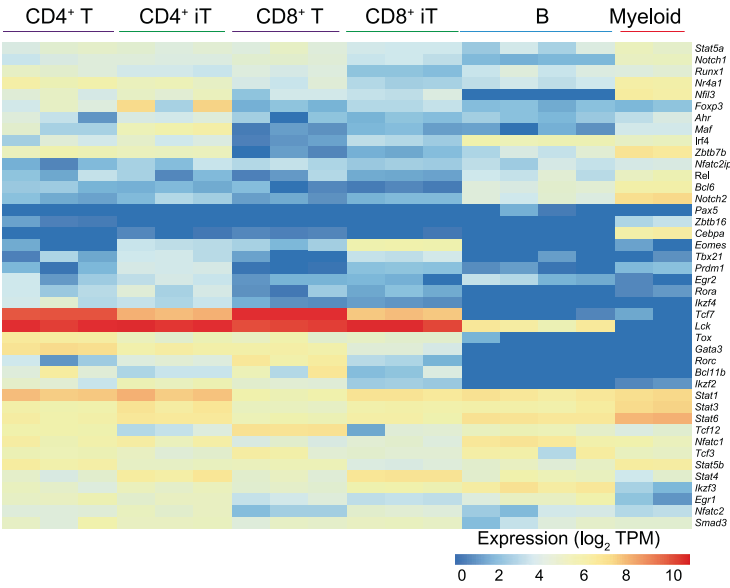

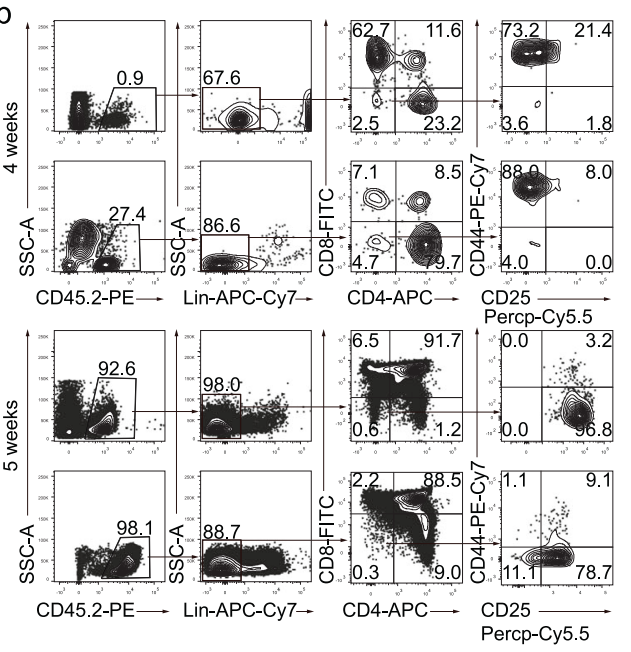
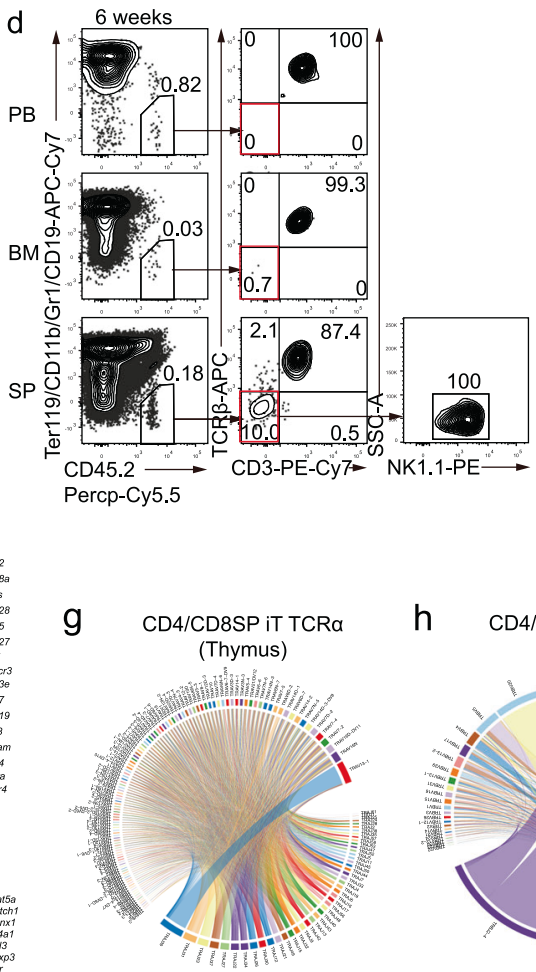

h $\quad$ CD4/CD8SP iT TCR $\beta$

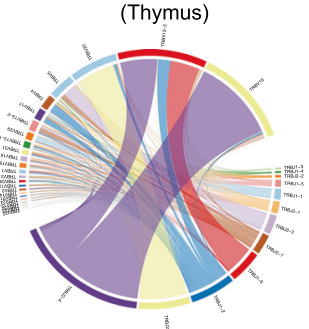

i $\quad$ CD4/CD8SP iT TCR $\alpha$ (Spleen)

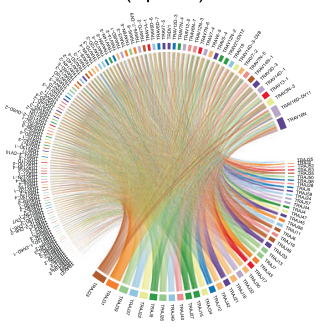

CD4/CD8SP iT TCR $\beta$

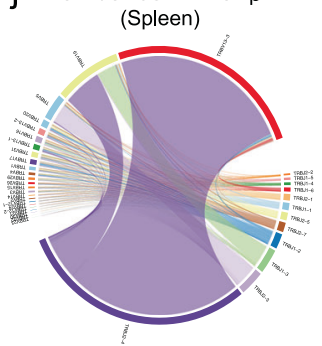

iT cell transfer, we transplanted allogeneic skin from BALB/c mice into the iT-Rag $1^{-1-}$ mice. The allogeneic skin grafts were rapidly rejected by $\mathrm{iT}$-Rag $1^{-1-}$ mice at around day 9 after transplantation, as indicated by bulged, ulcerative and necrotic lesions at the graft sites (Fig. 5a). Besides the mature iT cells (CD4SP, CD8SP) in the PB of iT-Rag $1^{-1-}$ mice (Fig. 5b), activated CD4SP and CD8SP iT cells
$\left(\mathrm{CD} 44^{\text {high }} \mathrm{CD} 9^{+}\right)$were also detected in the rejected allogeneic skin tissues (Fig. $5 \mathrm{C}$ ). The iT-Rag $1^{-1-}$ mice still showed the existence of iT cells in PB thirty days after the primary allogeneic rejection, and again rejected the secondary allogeneic skin grafts (Supplementary information Fig. S6). Flow cytometry indicated that IL $17^{+}$and IFN $\gamma^{+} \mathrm{CD}^{+}$iT cells, and IFNY ${ }^{+} \mathrm{CD}^{+} \mathrm{iT}$ cells existed 
Fig. 2 Tissue distributions, transcriptome characterization, and TCR $\alpha / \beta$ diversities of ESC-derived T Cells. a Flow cytometry analysis of mature iT cells in spleen (SP), lymph node (LN), and PB of B-NDG mice transplanted with ESC-derived hematopoietic cells. Each B-NDG mouse was transplanted with one million iHPCs collected at day 21. Representative mouse was sacrificed and analyzed at 5 and 6 weeks after transplantation. Data from two representative mice are shown. b Flow cytometry analysis of iDN cells in the thymus of B-NDG mice transplanted with ESC-derived hematopoietic cells. Each B-NDG mouse was transplanted with one million iHPCs at day 21. Representative mouse was sacrificed and analyzed at 4 and 5 weeks after transplantation. Data from four representative mice of two independent experiments are shown. Lin was defined as Ter119- $\mathrm{CD} 11 \mathrm{~b}^{-} \mathrm{Gr} 1^{-} \mathrm{CD} 19^{-} \mathrm{B} 220^{-} \mathrm{NK} 1.1^{-} \mathrm{TCR} \gamma \delta^{-}$. c Flow cytometry analysis of iHPC derivatives in bone marrow (BM). Each B-NDG mouse was transplanted with one million iHPCs collected at day 10 in the presence of OP9-DL1 feeder cells. Representative mouse was sacrificed and analyzed 5 weeks and 6 weeks after transplantation. The BM-derived iHPCs (CD45.2 ${ }^{+}$Lin ${ }^{-} \mathrm{c}^{-}$ kit $^{\text {mid }} \mathrm{Sca}^{+}$) were sorted for the 2 nd transplantation. Data from two mice are shown. d Flow cytometry analysis of iT and iNK cells in PB, spleen (SP) and bone marrow (BM) 6 weeks after the 2nd transplantation. Five hundred LSK cells from primary iT mice were used as input for secondary transplantation. The secondary recipients were sacrificed and analyzed 6 weeks after transplantation. Data from one mouse are shown. e Characterization of surface markers on CD4SP and CD8SP iT cells. CD4SP and CD8SP iT cells were sorted from the spleens of B-NDG mice transplanted with ESC-derived hematopoietic cells at week 5. One biological replicate per column. Myeloid cells ( $n=2$ sample repeats):

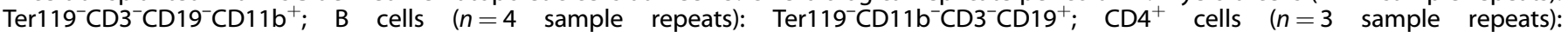

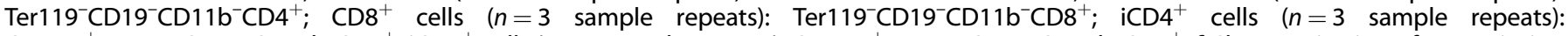
CD45.2 $2^{+}$Ter $119^{-} \mathrm{CD} 19^{-} \mathrm{CD} 11 \mathrm{~b}^{-} \mathrm{CD} 4^{+} ;$;CD8 ${ }^{+}$cells $\left(n=3\right.$ sample repeats): CD45.2 $2^{+}$Ter $119^{-} \mathrm{CD} 19^{-} \mathrm{CD} 11 \mathrm{~b}^{-} \mathrm{CD} 8^{+}$. f Characterization of transcription factors in CD4SP and CD8SP iT cells. $\mathbf{g}$ Chord diagram of TCR $\alpha$ diversity in thymus iT cells. $\mathbf{h}$ Chord diagram of TCR $\beta$ diversity in thymus iT cells. i Chord diagram of TCR $\alpha$ diversity in spleen iT cells. $\mathbf{j}$ Chord diagram of TCR $\beta$ diversity in spleen iT cells. Aliquots of sorted 15,000 naïve CD4SP and CD8SP iT cells from either thymus or spleen of iT-B-NDG mice were used as cell inputs for TCR $\alpha \beta$ sequencing

a

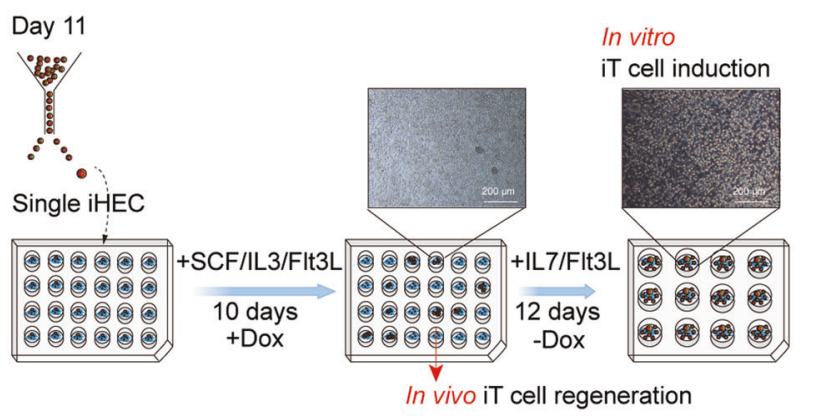

C

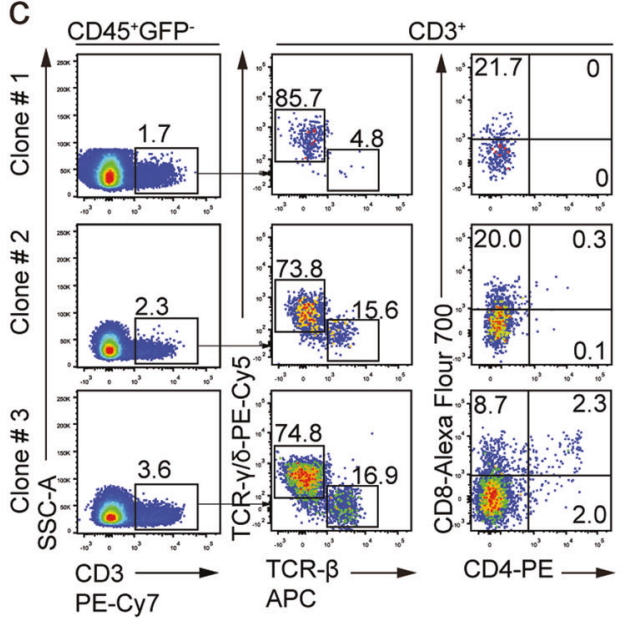

b

\begin{tabular}{ccll}
\hline Experiments & $\begin{array}{l}\text { Hematopoietic colony } \\
\text { forming efficiency } \%\end{array}$ & $\begin{array}{l}\text { iT induction } \\
\text { (in vitro) } \%\end{array}$ & $\begin{array}{l}\text { iT regeneration } \\
\text { (in vivo) } \%\end{array}$ \\
\hline I & $20.8(20 / 96)$ & $100(10 / 10)$ & $30(3 / 10)$ \\
II & $15.6(15 / 96)$ & $100(5 / 5)$ & NA \\
III & $19.8(19 / 96)$ & NA & $30(3 / 10)$ \\
IV & $22.9(22 / 96)$ & $100(15 / 15)$ & $20(1 / 5)$ \\
\hline
\end{tabular}

d

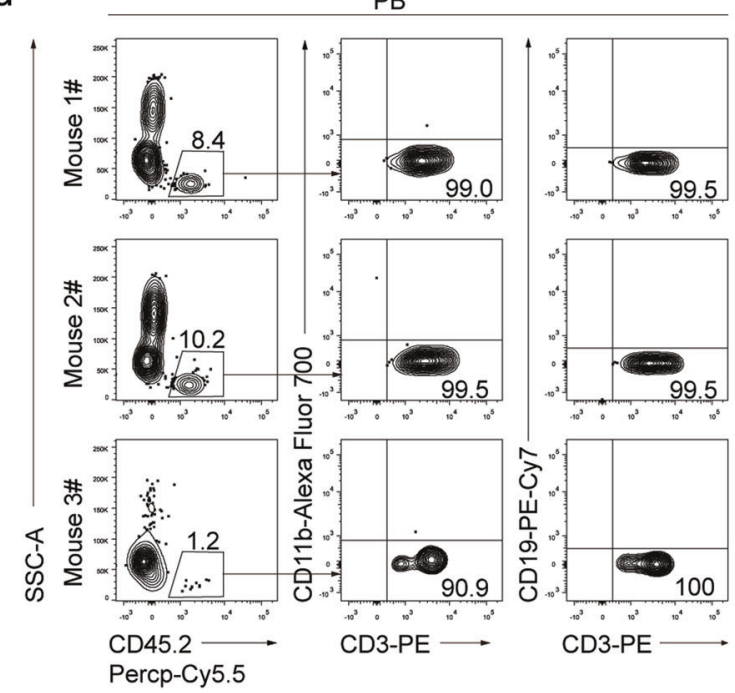

Fig. 3 Assessment of T potential of single iHECs from iR9-ESC. a The strategy of T cell induction from iR9-ESC-derived single iHECs. Single iHECs were sorted into individual wells (24-well plates) pre-seeded with OP9-DL1 feeder cells (10,000 cells/well) $12 \mathrm{~h}$ prior maturation in EM medium with doxycycline $(1 \mu \mathrm{g} / \mathrm{mL})$. Doxycycline was sustained for 10 days during the maturation step. After maturation, the bulk blood cells were assessed for $\mathrm{T}$ lineage generation potential. For in vivo $\mathrm{T}$ cell regeneration, the single iHEC-derived bulk hematopoietic cells (day 10) were transplanted into individual B-NDG recipients. For in vitro T cell induction, the medium was changed to T cell induction medium (TIM, $\alpha$ MEM, 20\% DFBS, and 1\% GlutaMAX) supplemented with $2 \%$ conditioned medium derived from supernatants of AFT024-hFlt3L and AFT024hIL7 cell culture for sustaining 12 days. b Single iHECs efficiently gave rise to T cells. Three hundred and eighty-four single iHECs at day 11 were sorted into individual wells ( 24 well plates). Thirty single-iHEC-formed blood colonies were induced for T cell generation in vitro. Cell collections of Twenty-five single-iHEC-formed blood colonies were transplanted into 25 individual B-NDG mice for the assessment of T lymphopoiesis in vivo. c Flow cytometry analysis of induced T cells from in vitro induction of single iHECs. iT cells from single iHEC culture product (day 22) were analyzed. Plots of iT cells induced from one representative colony are shown. $\mathbf{d}$ Single iHEC-derived hematopoietic cells gave rise to mature iT cells in PB of B-NDG recipient mice 4 weeks after transplantation. Plots of one representative mouse are shown 
a
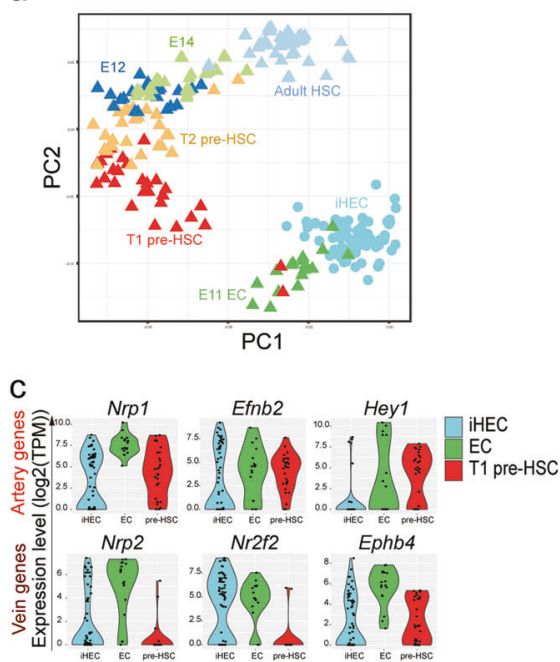

b EC HEC T1 pre-HSC

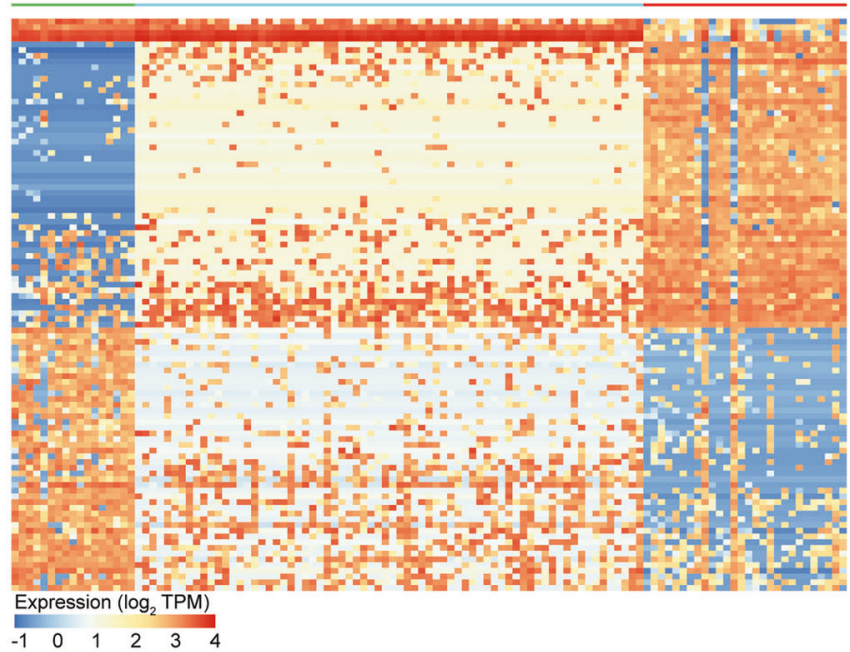

e

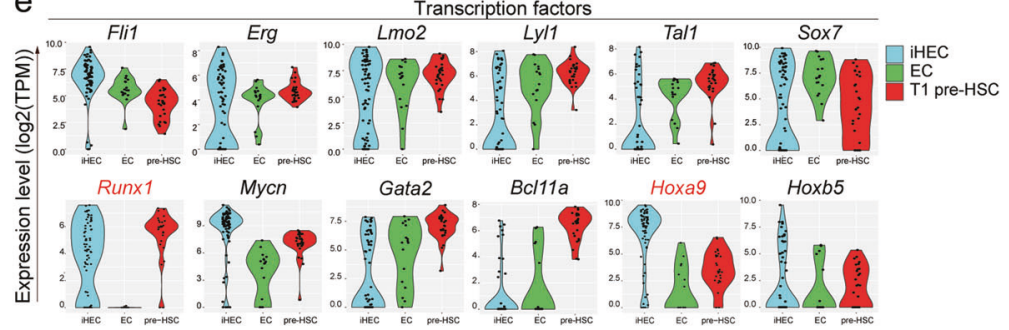

g
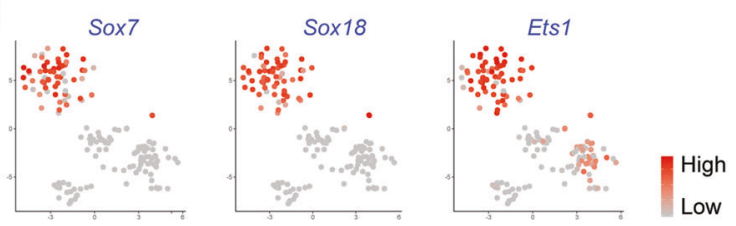

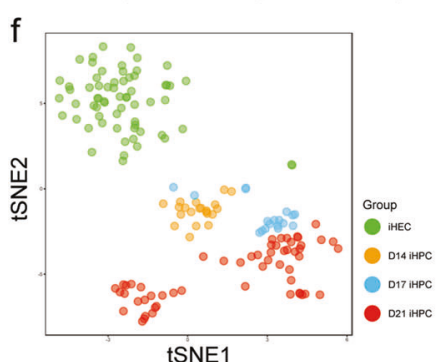

h

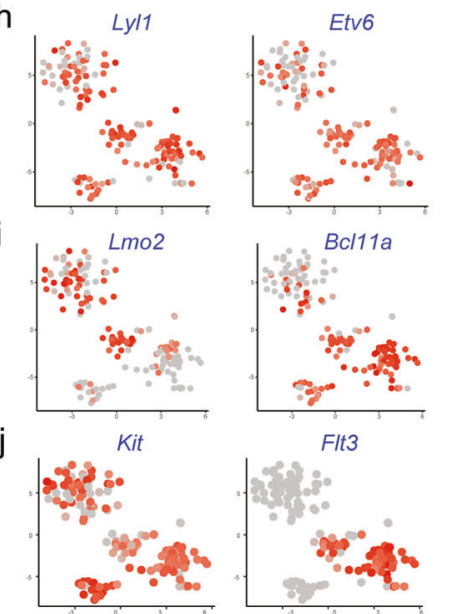

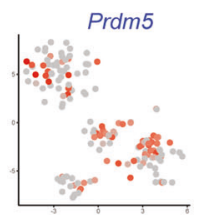

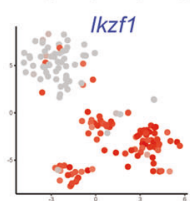

$C d 7$

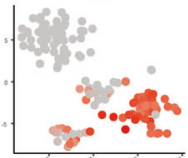

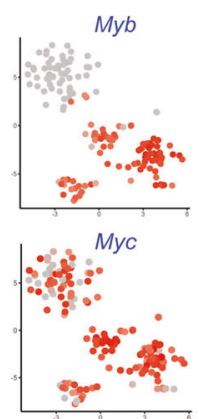

Cor9

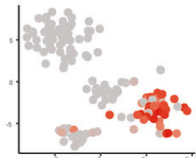

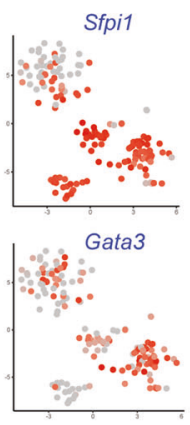

Ccr7

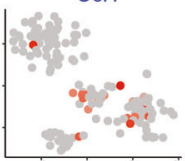

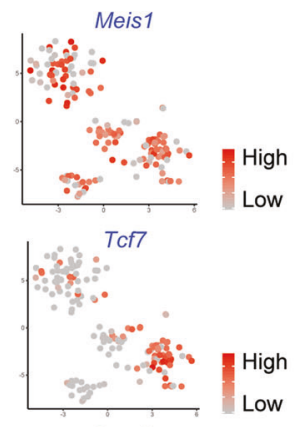

Cxcr4

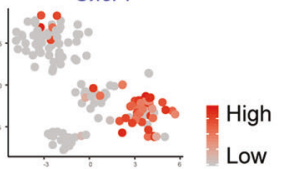

in the primary- and secondary-rejected skin grafts (Fig. 5d). Collectively, these results indicated that the adoptively transferred iT cells in $R a g 1^{-1-}$ mice mediated rejection of allogeneic skin grafts and sustained immunological memory, suggestive of a typical adaptive immune response.
The iT cells derived from TCR-edited iPSCs eradicate tumor cells in vivo

Given the advantages of unlimited cell source and gene-editing advantage of iPSC, we introduced tumor antigen-specific TCR (MHC-I-restricted OVA TCR, OT1) into iR9-iPSCs and further 
Fig. 4 Single-cell transcriptomic characterization of iHECs and iHPCs. a Principal component analysis (PCA) of iHECs and developmental E11 AGM-derived ECs, T1 pre-HSCs, T2 pre-HSCs, E12 HSCs, E14 HSCs, and adult HSCs. TPM values of genes in iHECs $(n=70)$, natural E11 AGMderived ECs $(n=17)$, T1 pre-HSCs $(n=28)$, T2 pre-HSCs $(n=32)$, E12 HSCs $(n=21)$, E14 HSCs $(n=32)$ and adult HSCs $(n=47)$ from single-cell RNA-Seq data were calculated with Stringtie package. b The expression of the top 100 genes contributing most to PC2 (50 genes for each direction). The expression value (TPM) of each gene was converted by log 2 and illustrated by pheatmap (R package). One column represents one cell repeat. c Violin plots show the expression profile of selected artery (A)- and vein (V)-related genes (A: Nrp1, Efnb2, and Hey1; V: Nrp2, $\mathrm{Nr} 2 \mathrm{f2}$, and Ephb4) in single iHECs. The expression value (TPM) of each gene was converted by log2 and illustrated by ggplot2 (R package). One point represents one cell. d Violin plots show the expression profile of selected surface markers (Cdh5, Esam, Tek, Procr, Cd47, and Cd63) in single iHECs. The expression value (TPM) of each gene was converted by log2 and illustrated by ggplot2 (R package). One point represents one cell. e Violin plots show the expression profile of selected transcription factors (Fli1, Erg1, Lmo2, Lyl1, Tal1, Sox7, Runx1, Mycn, Gata2, Bcl11a, Hoxa9, and Hoxb5) related to hematopoietic development in single iHECs. The expression value (TPM) of each gene was converted by log2 and illustrated by ggplot2 (R package). One point represents one cell. f Two-dimensional t-SNE analysis of iHEC and iHPC single-cell RNA-Seq. For single-cell RNA-Seq, the iHECs were collected at day 11, and the iHPCs were collected at day 14, 17 and 21 . Each dot represents one cell. The TPM values of genes in iHECs $(n=65)$, iHPCs at day $14(n=21)$, day $17(n=18)$ and day $21(n=56)$ from single-cell RNA-Seq data were calculated with Stringtie package. Cell types were defined as: (1) iHEC, CD31 ${ }^{+} \mathrm{CD} 41^{\text {low }} \mathrm{CD} 45^{-}$c-kit ${ }^{+} \mathrm{CD} 201^{\text {high }}$; (2) day-14 and day-17 iHPC, CD45 ${ }^{+}$Lin(Ter119/Gr1/F4-80/CD2/CD3/CD4/CD8/CD19/FceRlo) ${ }^{-}$; (3) day-21 iHPC, Ter119 CD45 $^{+}$c-kit ${ }^{+}$CD127 ${ }^{+}$. g t-SNE analysis of the expression pattern of selected endothelia-related transcription factors (Sox7, Sox18, and Ets1) in iHECs and iHPCs. h t-SNE analysis of the expression pattern of selected hematopoiesis-related transcription factors (Lyl1, Etv6, Prdm5, Myb, Sfpi1, and Meis1) in iHECs and iHPCs. i t-SNE analysis of the expression pattern of selected T cell development-related transcription factors (Lmo2, Bcl11a, Ikzf1, Myc, Gata3, and Tcf7) in iHECs and iHPCs at day 14, day 17, and day 21. j t-SNE analysis of the expression pattern of selected lymphopoiesis-related surface proteincoding genes (Kit, Flt3, Cd7, Ccr9, Ccr7, and Cxcr4) in iHECs and iHPCs at day 14, day 17, and day 21

assessed the anti-tumor activity of the derived OT1 iT cells. We reprogrammed mouse MEFs (C57BL/6 background, CD45.2 strain) into iPSCs using retroviruses carrying Oct4/KIf4/Sox2. Two cassettes of rtTA-TRE-Runx1-p2a-Hoxa9-HygroR and CAG-OT1-TCRIRES-GFP-PuroR were inserted into the loci of Rosa26 and Hipp11 of iPSC (OT1-iR9-iPSC), respectively (Fig. 6a). Intracellular staining indicated that the OT1-TCR was expressed in the OT1-iR9-iPSCs (Fig. 6b). The OT1-iR9-iPSCs were further induced into OT1-iHECs (Fig. 6c) and OT1-iHPCs (Fig. 6d). We transplanted the OT1-iHPCs ( 3 million/mouse) into irradiated $(4.5 \mathrm{~Gy}) \mathrm{Rag} 1^{-/-}$mice (OT1-iHPC recipients) to reconstitute OT1-iT lymphopoiesis. Six weeks after transplantation, $\mathrm{GFP}^{+} \mathrm{CD}^{+}$iT cells expressing OT1 TCRa $\beta$ were detected in PB of the OT1-iHPC recipients (Fig. 6e). We then engrafted E.G7-OVA tumor cells into the groin of the $\operatorname{Rag}^{-1-}$ or OT1-iT-reconstituted $R a g 1^{-/-}$mice (OT1-iT-Rag $1^{-1-}$ mice) by subcutaneous injection (0.2 million/mouse). Tumor growth kinetics demonstrated that the E.G7-OVA tumors were dramatically inhibited in the OT1-iT-Rag $1^{-/-}$mice in comparison with the control Rag1 $1^{-/-}$mice (Fig. 6f). We sacrificed the OT1-iT-Rag ${ }^{-1-}$ mice for the distribution analysis of the iT cells in tumors and lymphoid organs 19 days after the tumor cell transplantation. Flow cytometry analysis demonstrated that the E.G7-OVA tumors in the OT1-iT-Rag $1^{-/-}$mice were infiltrated with $\mathrm{CD}^{+}$OT1-iT cells, which contained effector $\left(\mathrm{CD} 44^{+} \mathrm{CD}_{22} \mathrm{~L}^{-}\right)$and memory $\left(\mathrm{CD} 44^{+} \mathrm{CD} \mathrm{L}^{+}\right)$iT cells, and IFNy-secreting iT cells (Fig. 6g). We also observed abundant $\mathrm{CD} 8^{+}$iT cells carrying OT1 TCRa $\beta$ in the bone marrow, lymph node, and spleen of these mice (Supplementary information Fig. S7). Collectively, these data indicate that the iT cells derived from TCR-engineered iPSCs show anti-tumor activity in a solid tumor model.

\section{DISCUSSION}

In this study, the iHECs derived from iR9-PSCs gave rise to blood progenitor cells preferentially differentiating into iT cells in vivo. It is possible that the combinatory expression of Runx 1 and Hoxa9, pivotal transcription factors for definitive hematopoiesis ${ }^{22-24,63}$ and $\mathrm{T}$ cell development, ${ }^{18}$ synergistically and preferentially orchestrates the $\mathrm{T}$ and NK lineage potentials but intrinsically compromises the other blood lineage potentials during the early EHT and subsequent hematopoietic maturation phases in our induction protocol. Given the developmental evidence that an earlier wave of hematopoiesis preceding HSC emergence also produces blood progenitors possessing the $\mathrm{T}$ cell lineage potential, ${ }^{14-16}$ it is also possible that the iR9-PSC-derived iHPCs resemble the developmental HPCs prior to the occurrence of definitive HSCs since overexpression of Runx1 and Hoxa9 at definitive HSC phase promoted myeloid-instead of lymphoidbiased hematopoiesis in vivo (Supplementary information Fig. S8). The hematopoietic maturation step in the presence of OP9-DL1 feeder line unlikely causes T-lineage-biased iHPCs, as an inducible expression of another transcription factor cocktail in PSCs exactly using the same protocol gave rise to iHPCs preferentially contributing to B lymphopoiesis in B-NDG recipients (unpublished data). Nonetheless, our data support the concept that synergies of distinct transcription factors intrinsically determine variable hematopoietic lineage potentials at as early as hemogenic endothelial cell stage.

Intravenous infusion of the iHPCs derived from iR9-PSCs successfully reconstituted iT lymphopoiesis in vivo. The induced LSK cells from the primary iHPC recipients further gave rise to T lymphocytes in secondary recipients. The occurrence of iDN1, iDN2, iDN3, iDN4 cells at different time-points in the thymi of iTB-NDG mice strongly indicate that the induced pre-thymic progenitors ( $\mathrm{Lin}^{-} \mathrm{C}-\mathrm{kit}^{+} \mathrm{CD} 127^{+} / \mathrm{CD} 135^{+}$) have the capacities of homing to central lymphoid organs and developing normally following a cellular trajectory resembling natural T cell development. Despite the inefficient generation of CD4SP iT cells in vitro due to the MHC-I-restricted OP9-DL1 feeder cells, robust phenotypic CD4SP iT cells generated in vivo and successful allogeneic rejection mediated by the CD4SP and CD8SP iT cells support that the regenerated regulatory iT cells possess normal immune functions. In combination with the new method of generating induced $B(i B)$ cells (unpublished data), it would be promising to further test the coordinated immune responses of iT cells and iB cells in infection models. Besides the pivotal roles of exogenous Runx1 and Hoxa9 during EHT and subsequent iHPC maturation phases, we could not exclude the possibilities that the weak leaky expression of these two factors further facilitated the iT cell development in vivo after infusion into immune-deficient mice, as Runx 1 and Hoxa9 are also involved in T cell development in bone marrow ${ }^{64}$ and thymus. ${ }^{18}$ In contrast to our approach, an induced T cell progenitor population (DN2/ DN3 cell phase) from mouse ESC lacked thymus-homing capacity in vivo and required congenic fetal thymus organ for further development into mature T cells, ${ }^{6}$ which implicated that an intrinsic gene network program essential for physiological $\mathrm{T}$ cell development were not fully activated during hematopoietic induction from PSCs, which can be rescued by exogenous expression of Runx1 and Hoxa9. Nonetheless, our approach fully reconstitutes functional $\mathrm{T}$ lymphopoiesis in vivo using PSC source, which avoids the malfunction risks of in vitro- 
a
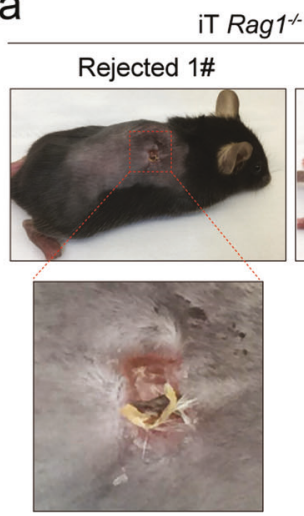

Rag1- (Day 30)

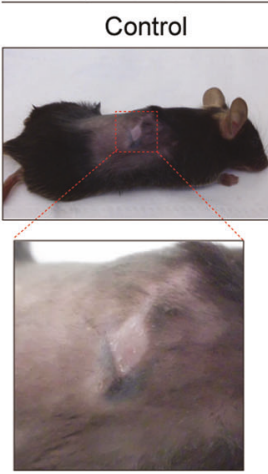

b

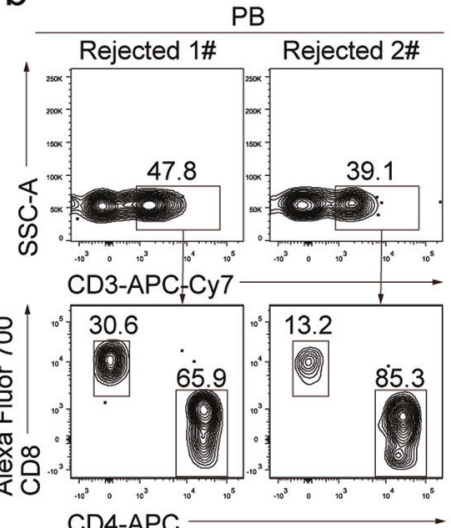

C

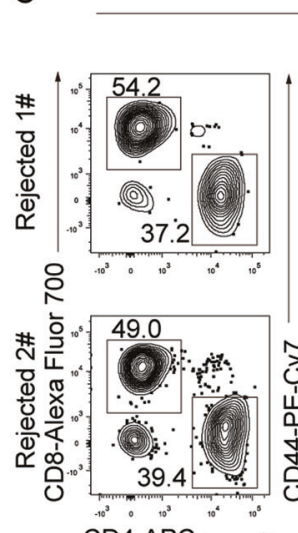

CD4-APC $\longrightarrow$
Rejected skin

$\mathrm{CD}^{+}$
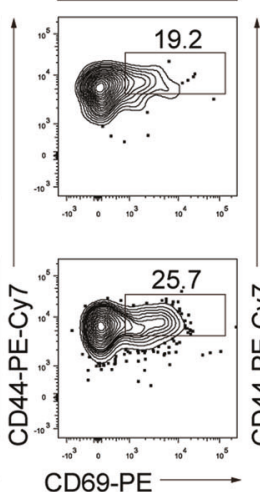
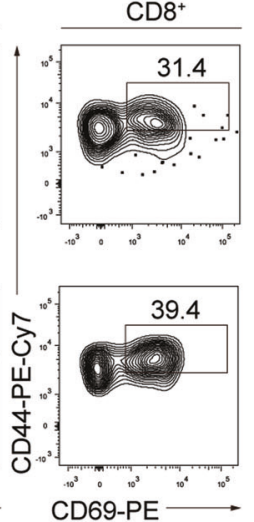

d
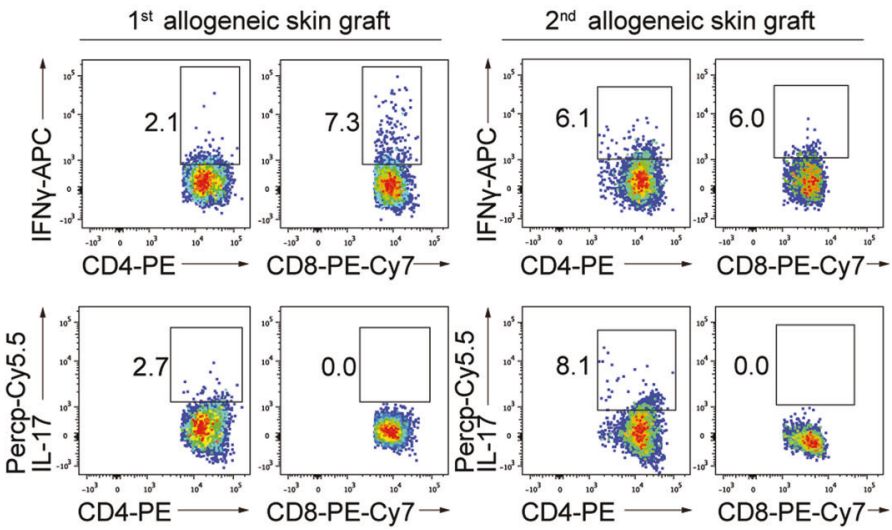

Fig. 5 Adoptively transferred iT cells reject allogeneic skin in $R a g 1^{-1-}$ mice. a The images of allogeneic skin grafts. Representative images of rejected allogeneic skin tissues on ESC-iT-Rag $1^{-1-}$ (day 9) mice $(n=2)$ and grafted skin tissue on control Rag $1^{-1-}$ mice (day 30) were shown. $\mathbf{b}$ Flow cytometry analysis of the adoptively transferred ESC-iT cells in peripheral blood (PB) of Rag $1^{-1-}$ recipients 9 days after the allogeneic skin was grafted. Plots of two representative mice are shown. c Flow cytometry analysis of the activation status of the ESC-iT cells in the rejected allogeneic skin tissues. The rejected allogeneic skin tissues were from the adoptively ESC-iT cell-transferred Rag $1^{-1-}$ recipients 9 days after the allogeneic skin grafted. The activated ESC-iT cells were defined as $C D 4^{+} / C D 8^{+} C D 44^{\text {high }} C D 69^{+}$. Rejected skin tissues from two representative ESC-iT cell-transferred Rag $^{-1-}$ mice were analyzed. d Flow cytometry analysis of the intracellular cytokines IFN $\gamma$ and IL-17 secreted by the $\mathrm{CD}^{+}{ }^{+}$or $\mathrm{CD} 8^{+}$ESC-iT cells in rejected allogeneic skin tissues. The 1 st allogeneic skin grafts were analyzed at day 9 and the 2 nd allogeneic skin grafts were analyzed at day 6 after skin transplantation. Data from the primary and secondary rejected skin tissues from one representative ESC-iT cell-transferred $\operatorname{Rag}^{1-1-}$ mouse are shown

generated $\mathrm{T}$ cells due to the insufficiency of negative and positive selections.

The single iHECs exhibited a transcriptome signature resembling E11 AGM (aorta-gonad-mesonephros) ECs and pre-HSCs. Activating the signature genes lacking in the iHECs but abundant in natural E11 AGM ECs or pre-HSCs might further promote the production of a homogenous iHEC population, thus consequently resulting in more efficient $T$ cell generation or multi-lineage hematopoietic reconstitution. The feature of $\mathrm{T}$ cell-lineage-bias commitment from iR9-PSCs brings advantages for gene editing using iR9-PSCs rather than using canonical adult HSPCs, since manipulating HSPCs in vitro always faces stemness loss and might even introduce unknown impacts on the functions of other blood lineage derivatives from the edited HSPCs.

In conclusion, this study establishes a novel approach of preferentially reconstituting functional and therapeutic T lymphopoiesis in vivo using PSC source by defined transcription factors. At single cell resolution, we unveil that the T-lineage specification is determined at as early as hemogenic endothelial cell stage and identify the bona fide pre-thymic progenitors with thymushoming features. Given the enormous demand of regenerative $T$ lymphopoiesis in treating $\mathrm{T}$ cell-deficient and cancer-bearing patients, this study provides insight into therapeutic T lymphopoiesis using PSC source.

\section{MATERIALS AND METHODS}

Mice

B-NDG (NOD-Prkdc $c^{S c i d} / L 2 r g^{t m 1} /$ Bcgen, $C D 45.1^{+}$) mice were purchased from Biocytogen Jiangsu Co., Ltd (Jiangsu, China). BALB/C and C57BL/6 $\left(\mathrm{CD} 45.2^{+}\right)$mice were purchased from Bejing Vital River Laboratory Animal Technology. Rag $1^{-/-}$mice (C57BL/6 background) were a gift from Dr. Zhihua Liu from the Institute of Biophysics (CAS, China). Mice were housed in the SPF-grade animal facility of the Guangzhou Institutes of Biomedicine and Health, Chinese Academy of Sciences (GIBH, CAS, China). All animal experiments were approved by the Institutional Animal Care and Use Committee of Guangzhou Institutes of Biomedicine and Health (IACUC-GIBH).

\section{Cell culture}

Mouse embryonic fibroblasts (MEFs) were derived from 13.5 d.p.c C57BL/6 mouse embryos. MEFs were maintained in DMEM/high glucose (Hyclone), 10\% FBS (Natocor) supplemented with $1 \%$ 


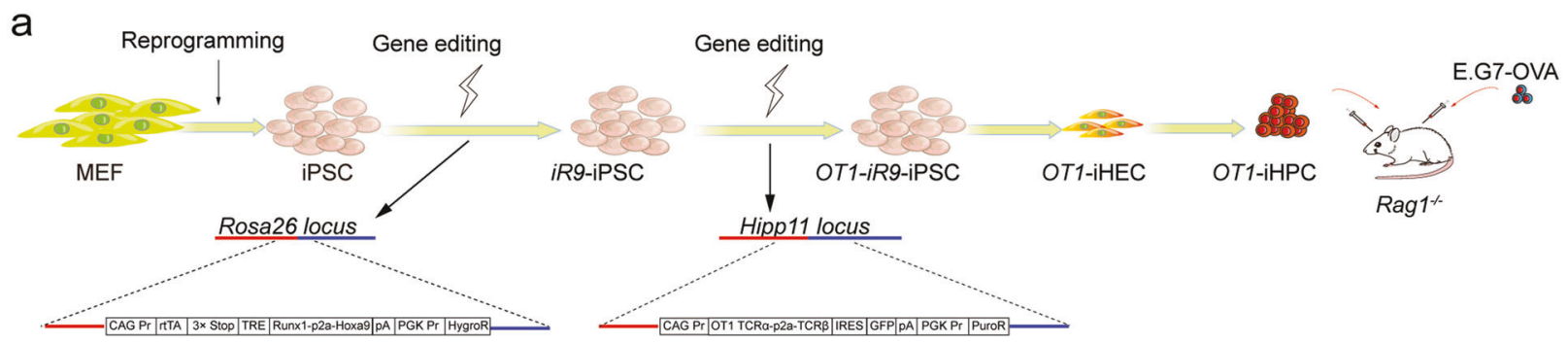

b

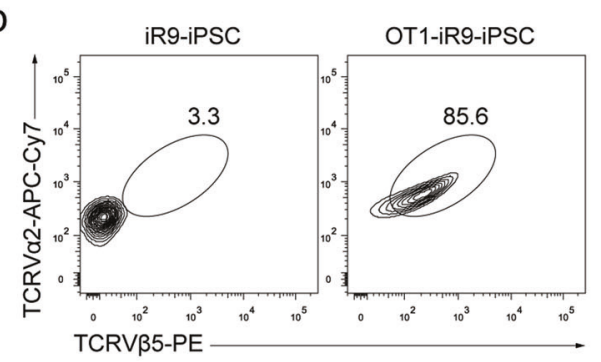

C

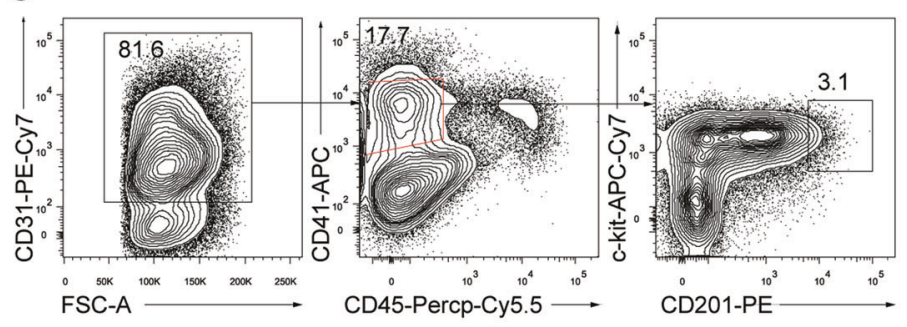

d
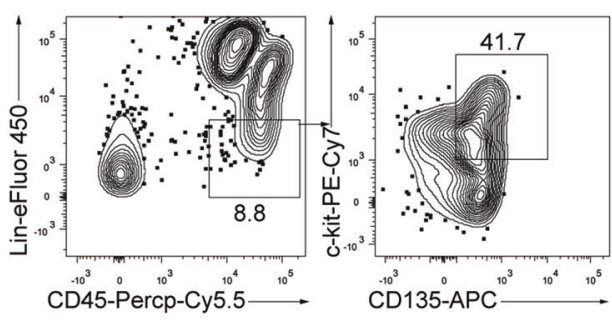

$f_{400}$

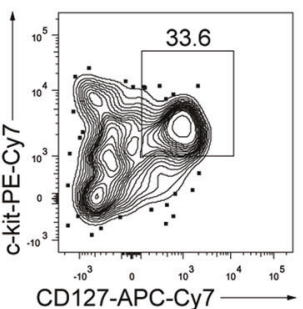

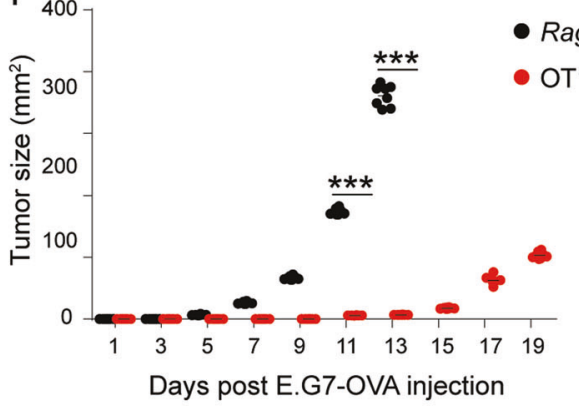

e

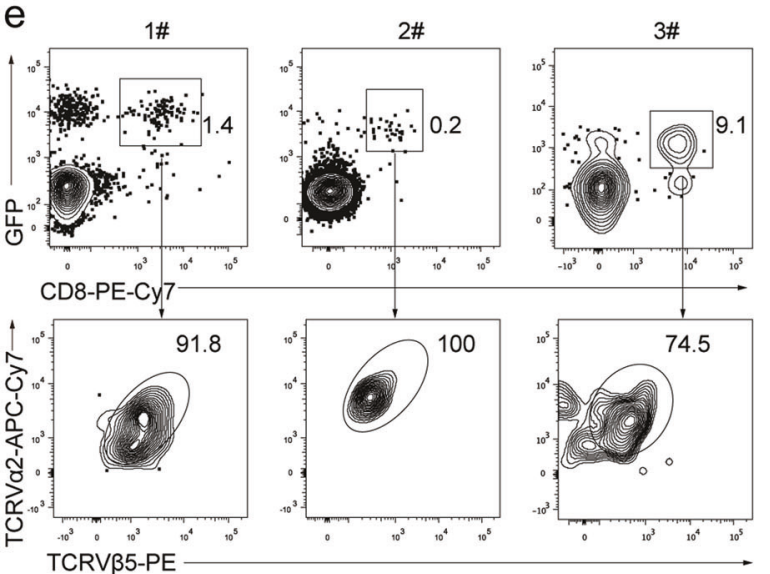

g
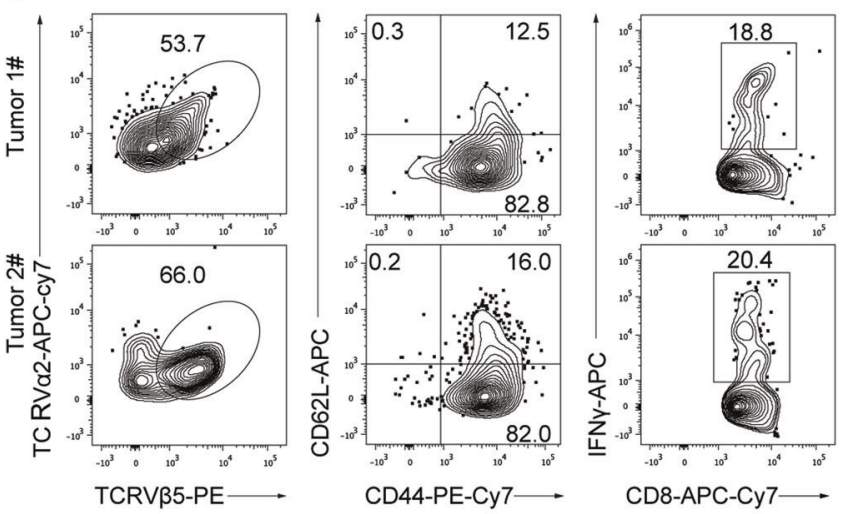

nonessential amino acids (NEAA, Gibco). C57BL/6 mouse embryonic stem cells (Biocytogen) were maintained on feeder layers in ES medium containing DMEM/high glucose, 15\% FBS (Gibco), 1\% NEAA, 1\% GlutaMAX (Gibco), 1\% Sodium Pyruvate (Gibco), $0.1 \mathrm{mM}$ $\beta$-mercaptoethanol (Gibco), $1 \mu \mathrm{M}$ PD0325901 (Selleck), $3 \mu \mathrm{M}$ Chir99021 (Selleck) and $1000 \mathrm{U} / \mathrm{mL}$ LIF. The OP9-DL1 cells (GFP ${ }^{+}$) were maintained in a-MEM (Gibco) supplemented with $20 \%$ FBS (CellMax). The AFT024 cell lines (ATCC) were maintained in DMEM/ high glucose, $10 \%$ FBS (Natocor) supplemented with $0.1 \mathrm{mM} \beta$ mercaptoethanol and 1\% Sodium Pyruvate. HEK293T (ATCC) and Plat-E (Cell Biolabs, Inc) cells were maintained in DMEM/high glucose supplemented with 10\% FBS (Natocor). E.G7-OVA cell line (ATCC) was cultured in RPMI 1640 (Gibco) supplemented with $10 \%$ FBS (Natocor), $1 \%$ GlutaMAX, 1\% sodium pyruvate, and $0.1 \mathrm{mM} \beta$ mercaptoethanol.
Hematopoietic differentiation

PSCs were trypsinized by $0.05 \%$ Trypsin-EDTA (Gibco) and resuspended in the basic differentiation medium (BDM: IMDM, $15 \%$ FBS (Gibco), $200 \mu \mathrm{g} / \mathrm{mL}$ iron-saturated transferrin (Sigma), $0.45 \mathrm{mM}$ monothiolglycerol (Sigma), $1 \%$ GlutaMAX, and $50 \mu \mathrm{g} / \mathrm{mL}$ ascorbic acid (Sigma)). To remove the feeder layers, the PSCs were plated into the $0.1 \%$ gelatin-coated (Merck Millipore) well, and the floating cells were collected after $40 \mathrm{~min}$. For embryoid body (EB) generation, ${ }^{65}$ the PSCs were resuspended at 100,000 cells $/ \mathrm{mL}$ in the BDM supplemented with $5 \mathrm{ng} / \mathrm{mL}$ BMP4 (Peprotech) and plated at $20 \mathrm{uL} /$ drop for inverted culture in $15 \mathrm{~cm}$ dishes. At day 2.5, EBs were replanted into gelatinized plates in BDM supplemented with $5 \mathrm{ng} / \mathrm{mL}$ BMP4 and $5 \mathrm{ng} / \mathrm{mL}$ VEGF (Peprotech). At day 6 , the medium was changed to BDM supplemented with $2 \%$ conditioned medium derived from the supernatants of AFT024- 
Fig. 6 OT1-iT cell therapy suppresses the solid tumor growth in mice transplanted with E.G7-OVA cells. a Schematic diagram of the generation of OT1-engineered iT cells for anti-tumor therapy. Mouse MEFs were isolated from CD45.2 ${ }^{+}$C57BL/6 mouse and reprogrammed into iPSCs with retroviruses expressing Oct4, KIf4, and Sox2. Then an rtTA-TRE-Runx1-Hoxa9-HygroR DNA cassette was inserted into the Rosa26 locus. Next, a CAG-OT1-IRES-GFP-PuroR expression element was inserted into the Hipp11 locus of iR9-iPSC. OT1-iR9-iPSC results in the production of CD8 ${ }^{+}$ T cells carrying TCRV $\alpha 2$ and TCRV $\beta 5$ (MHC class I-restricted, ovalbumin-specific TCR). OT1-iR9-iPSC-derived iHECs were induced into iHPCs (OT1-iHPCs) as described in the materials and methods section. The iHPCs were injected into irradiated (4.5 Gy) Rag $1^{-/-}$recipient mice (3 million/mouse, 8-10-week-old, C57BL/6 background). E.G7-OVA tumor cell line (C57BL/6 background) were transplanted into the groin of the $\operatorname{Rag}^{-1-}(n=8)$ or OT1-iT-Rag1 ${ }^{-1-}(n=8)$ mice by subcutaneous injection $(0.2$ million/mouse) 6 weeks after OT1-iHPC transplantation. b TCRV $\alpha 2$ and TCRV $\beta 5$ expression in OT1-iR9-iPSCs measured by intracellular staining. The iR9-iPSC was used as negative control. c Sorting gates of the OT1-iR9-iPSC-derived iHEC population at day 11 . The cells were enriched by streptavidin-beads recognizing biotin-CD31 before sorting. Representative plots from three independent experiments are shown. d Immuno-phenotypes of pre-thymic progenitors in iHPCs induced from OT1-iR9-iPSC-derived iHECs after ten-day maturation. Representative plots from three independent experiments are shown. Lin was

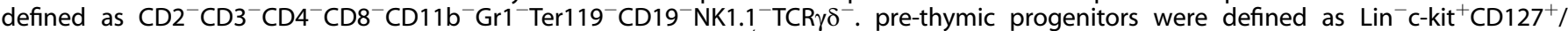
$\mathrm{CD}_{135^{+}}$. e TCRV $\alpha 2$ and TCRV $\beta 5$ expression of iT cells in PB of Rag $1^{-/-}$mice 6 weeks after transplantation of OT1-iR9-iPSC-derived iHPCs. Three representative mice from three independent experiments were analyzed. f Tumor growth in Rag $1^{-1-}$ and OT1-iT-Rag $1^{-1-}$ mice. E.G7-OVA cells were transplanted into the groin of the $\operatorname{Rag}^{-/-}(n=8)$ or OT1-iT-Rag $1^{-/-}$mice $(n=8)$ by subcutaneous injection (0.2 million/mouse). The length and width of the tumors were measured every other day by a caliper, and each tumor size was calculated as length $\times$ width (mm ${ }^{2}$ ). Mice with tumor size larger than $20 \mathrm{~mm}$ at the longest axis were euthanized for ethical consideration. ${ }^{* * *} P<0.001$ (independent $t$-test, twotailed). $\mathbf{g}$ Characterization of the OT1-iT cells in tumors. Tumors were isolated at day 19 after injection and disaggregated by collagenase IV to single cell suspensions. The effector iT cells were defined as CD44 ${ }^{+} \mathrm{CD} 62 \mathrm{~L}^{-}$. The memory iT cells were defined as CD44 ${ }^{+} \mathrm{CD} 62 \mathrm{~L}^{+}$. IFN $\gamma$ secreted by $\mathrm{CD}^{+}$OT1-iT cells in tumors were intracellular stained. Representative plots from two tumors are shown

mIL3, AFT024-mIL6, AFT024-hFlt3L and AFT024-mSCF cell culture. Doxycycline $(1 \mu \mathrm{g} / \mathrm{mL}$, Sigma) was added at day 6 . The medium was replaced every other day. The plates were seeded with OP9DL1 cells $(20,000$ cells/well, 12-well plate) $12 \mathrm{~h}$ prior to the hematopoietic maturation step in EM medium (a-MEM, 15\% DFBS (Hyclone), $200 \mu \mathrm{g} / \mathrm{mL}$ iron-saturated transferrin, $0.45 \mathrm{mM}$ monothiolglycerol, $1 \%$ GlutaMAX, $50 \mu \mathrm{g} / \mathrm{mL}$ ascorbic acid, $2 \%$ conditioned medium derived from supernatants of AFT024-mIL3, AFT024-hFlt3L and AFT024-mSCF cell culture and $1 \mu \mathrm{g} / \mathrm{mL}$ doxycycline). Then, $100-500$ sorted iHECs were seeded into each well for hematopoietic maturation. The EM medium was halfreplaced every two days.

Transplantation of iHPCs

Eight-ten-week-old B-NDG mice were sublethally irradiated (2.25 Gy) by an X-ray irradiator (RS2000, Rad Source Inc.), and 0.5-1 million PSC-derived iHPCs were injected into each irradiated BNDG mouse via retro-orbital veins. The mice were fed with water containing co-trimoxazole (Tianjin Lisheng Pharmaceutical co., LTD) for two weeks to prevent infection.

\section{T lymphocyte induction in vitro}

For T lymphocyte induction in vitro, OP9-DL1 coculture method ${ }^{1}$ was used with minor modifications. Briefly, the single-cell suspensions of iHPCs (day 21) were maintained on OP9-DL1 feeder cells in T cell induction medium (TIM, a-MEM, 20\% DFBS, and 1\% GlutaMAX) supplemented with $2 \%$ conditioned medium derived from supernatants of AFT024-hFlt3L and AFT024-hIL7 cell culture for sustained 12 days. The iHEC-derived cells were trypsinized into single-cell suspensions and replanted into fresh OP9-DL1 monolayers every 6 days. And the TIM was replaced every 3 days.

\section{Gene editing}

Mouse MEFs were reprogrammed into iPSCs as described. ${ }^{66}$ The CAG Pr-rtTA-3 $\times$ Stop-TRE-Runx1-p2a-Hoxa9-pA-PGK Pr-HygroR cassette was inserted into the Rosa26 locus of mouse ESC/iPSC. The positive clones (iR9-ESC/iPSC) selected by Hygromycin B $(150 \mu \mathrm{g} /$ $\mathrm{mL}$, Invivogen) were further cultured in ES medium supplemented with Dox $(1 \mu \mathrm{g} / \mathrm{mL})$. The induced expression of Runx 1 and Hoxa9 was confirmed by qPCR. For the generation of OT1-iR9-iPSC, a CAG Pr-OT1 TCRa-p2a-TCRß-IRES-GFP-PGK Pr-PuroR cassette was inserted into the Hipp11 locus of iR9-iPSC. The OT1 sequence was cloned from murine TCR OT1-2A.pMIG II (Addgene). The OT1iR9-iPSC-positive clones were further selected by Puromycin $(1 \mu \mathrm{g} /$ $\mathrm{mL}$, Invivogen) and the expression of OT1-TCR was measured by intra-cellular staining.
Flow cytometry and cell sorting

Single-cell suspensions were prepared by $0.05 \%$ Trypsin-EDTA and filtered by $70 \mu \mathrm{m}$ filter. Single cells were blocked by Fc (CD16/32) (93, eBioscience) antibody, and then stained with related antibodies. The following antibodies were used: c-kit (2B8, eBioscience), CD31 (390, eBioscience), CD41 (eBioMWReg30, eBioscience), CD45 (30-F11, eBioscience), CD45.1 (A20, eBioscience), CD45.2 (104, eBioscience), CD2 (RM2-5, eBioscience), CD3 (145-2C11, eBioscience), CD4 (GK1.5, eBioscience), CD8a (536.7, eBioscience), CD19(eBio1D3, eBioscience), B220 (RA3-6B2, eBioscience), CD11b (M1/70, eBioscience), NK1.1 (PK136, eBioscience), Ter119 (TER-119, eBioscience), Gr1 (RB6-8C5, eBioscience), CD201 (eBio1560, eBioscience), CD135 (A2F10, eBioscience), CD127 (A7R34 eBioscience), FceRla (MAR-1, biolegend), CD69 (H1.2F3, biolegend), CD62L (MEL-14, biolegend), IFNץ (XMG1.2, biolegend), IL17 (TC11-18H10.1, biolegend), CD44 (IM7, eBioscience), CD25 (PC61.5, eBioscience), TCR 3 (H57-597,

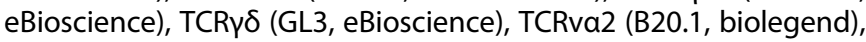
TCRvß5.1/5.2 (MR9-4, biolegend), Streptavidin PE-Cy7 (eBioscience), Streptavidin eFlour 450 (eBioscience), Streptavidin PE-Cy5 (biolegend). The cells were resuspended in the DAPI solution, or PI solution (eBioscience) and were analyzed with Fortessa cytometer (BD Biosciences). The cells were sorted using Arial II cytometer (BD Biosciences). The flow cytometry data were analyzed with FlowJo (Three Star, Ashland OR).

\section{Allogeneic skin transplantation}

Individual $\mathrm{Rag}^{-1-}$ mice (8-10 weeks old) were adoptively transferred with splenic cells equivalent to 5 million $\mathrm{CD}^{+}$and $\mathrm{CD}^{+}$iT cells from iT-B-NDG mice. Four days after iT cell transfer, the allogeneic skin (BALB/c background) was transplanted as described. ${ }^{67}$ Grafts were considered rejection if there was a loss of distinct border, visible signs of ulceration and necrosis to $80 \%$ of the graft area. The rejected skin tissues were removed for analysis 9 days after skin transplantation. To analyze activated iT cells in rejected skin grafts, the single-cell suspensions were prepared as described. ${ }^{68}$ The activated alloreactive iT lymphocytes were defined as CD45.2 $2^{+}$Ter119 ${ }^{-} \mathrm{CD} 11 \mathrm{~b}^{-} \mathrm{CD} 9^{+} \mathrm{CD} 44^{+} \mathrm{CD} 4^{+} / \mathrm{CD} 8^{+}$. To analyze cytokines released by the alloreactive iT cells, we used anti-IL17 and anti-IFNy antibodies following an intracellular staining protocol (eBioscience).

\section{OT1-iT anti-tumor assay}

For the reconstitution of the OT1-iT cells in $R a g 1^{-1-}$ mice, three million OT1-iHPCs were transplanted into each irradiated $\mathrm{Rag} 1^{-/-}$ mouse (4.5 Gy). OT1-iT cells $\left(\mathrm{GFP}^{+} \mathrm{CD}^{+} \mathrm{TCRV}^{\mathrm{T}} 5^{+} \mathrm{TCRV}^{+}{ }^{+}\right.$) in $\mathrm{PB}$ 
were analyzed six weeks post-transplantation. The E.G7-OVA cells were transplanted into the groin of the OT1-iT-reconstituted mice by subcutaneous injection ( $0.2 \mathrm{million} / \mathrm{mouse})$. The tumor size was measured every 2 days and was calculated as length $\times$ width $\left(\mathrm{mm}^{2}\right)$. Mice with tumor size larger than $20 \mathrm{~mm}$ at the longest axis were euthanized for ethical consideration. To analyze the tumorinfiltrating OT1-iT cells, tumors were isolated at day 15 and digested for $30 \mathrm{~min}$ at $37^{\circ} \mathrm{C}$ by collagen IV solution $(1 \mathrm{mg} / \mathrm{mL}$, Gibco) after being cut up. Then, the single-cell suspensions were harvested for staining. The activated iT cells were defined as $\mathrm{CD} 45.2^{+} \mathrm{GFP}^{+} \mathrm{CD}^{+} \mathrm{CD}^{+} 4^{+} \mathrm{CD} 2 \mathrm{~L}^{-}$.

RNA-seq and data analysis

The cDNA of single iHECs sorted at day 11, and iHPCs at day 14, 17 , and 21 or 1,000 CD4SP/CD8SP iT-cell aliquots from spleens of iT-B-NDG mice was generated and amplified using Discover-sc WTA Kit V2 (Vazyme). The quality of amplified cDNA was assessed by qPCR analysis of housekeeping genes (B2m and Gapdh). Samples that passed quality control were used for sequencing library preparation using TruePrep DNA Library Prep Kit V2 (Vazyme). All libraries were sequenced by illumina sequencer NextSeq 500. The raw data (fastq files) were generated using bcl2fastq software (version 2.16.0.10) and were uploaded to the Gene Expression Omnibus public database (GSE121371, GSE121373, GSE128738). The raw reads were aligned to mouse genome mm10 by HISAT2 (version 2.1.0) ${ }^{69}$ and the expression levels in TPM were estimated by StringTie (version 1.3.4). ${ }^{70,71}$ The wild-type CD4SP T cells, CD8SP T cells, myeloid cells, and B cells sequencing data (GSE105057) were downloaded from Gene Expression Omnibus. ${ }^{13}$ Heat maps were plotted using pheatmap (version 1.0.8). The natural embryonic single-cell data (endothelial cells $\left(\mathrm{CD} 31^{+} \mathrm{VE}\right.$-cadherin ${ }^{+} \mathrm{CD} 41^{-} \mathrm{CD} 43^{-} \mathrm{CD} 45^{-}$Ter $\left.119^{-}\right)$, T1 pre-

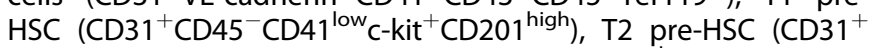

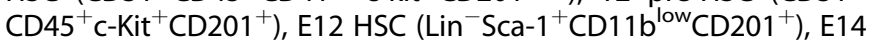
$\mathrm{HSC}\left(\mathrm{CD} 45^{+} \mathrm{CD} 150^{+} \mathrm{CD} 48^{-} \mathrm{CD} 201^{+}\right)$, and adult HSC $\left(\mathrm{CD} 45^{+}\right.$ $\left.\mathrm{CD} 150^{+} \mathrm{CD} 48^{-} \mathrm{CD} 201^{+}\right)$) were downloaded from Gene Expression Omnibus (GSE67120). ${ }^{35}$ The batch effects of single-cell data between iHECs and natural embryonic cells were removed using ComBat (sva R package, version 3.26.0). The prcomp function of stats (R package, version 3.4.4) was used for Principle Component Analysis (PCA). The DESeq2 was used for differential expression analysis. The PCA plot and violin plot were plotted using ggplot2 ( $R$ package, version 2.2.1). t-SNE was performed by Rtsne ( $R$ package version 0.15 ). The transcripts per million (TPM) values of transcription factors were log2-converted.

For TCRa $\beta$ sequencing, 15,000 sorted CD4SP, and CD8SP naïve iT cells were sorted from thymus or spleen of iT-B-NDG mice. The sorted iT cells of thymus were gated on CD45.2 Ter119 ${ }^{-} \mathrm{CD} 11 \mathrm{~b}^{-} \mathrm{Gr}^{-} \mathrm{CD}^{-} 9^{-} \mathrm{B} 220^{-} \mathrm{NK} 1.1^{-} \mathrm{TCR} \gamma \delta^{-} \mathrm{CD}^{+} \mathrm{CD}^{-}$and $\mathrm{CD} 45.2^{+}$Ter119- $\mathrm{CD} 11 \mathrm{~b}^{-} \mathrm{Gr}^{-} \mathrm{CD}_{19}^{-} \mathrm{B} 220^{-} \mathrm{NK} 1.1^{-} \mathrm{TCR} \gamma \delta^{-} \mathrm{CD}^{-} \mathrm{C}-$ $\mathrm{D} 8{ }^{+}$. The splenic naïve iT cells were gated on $\mathrm{CD} 45.2^{+} \mathrm{CD} 4^{+}$ $\mathrm{CD} 8{ }^{-} \mathrm{CD}_{22} \mathrm{~L}^{+} \mathrm{CD} 44^{-}$and $\mathrm{CD} 45.2^{+} \mathrm{CD}^{-} \mathrm{CD}^{+} \mathrm{CD}^{-} \mathrm{L}^{+} \mathrm{CD} 44^{-}$. The CDNA was generated and amplified by SMARTer Mouse TCRaß Profiling Kit (Clontech). Libraries were sequenced by illumina sequencer MiSeq $(2 \times 250$ cycles). The raw data (fastq files) were generated using illumina bcl2fastq software and were uploaded to Gene Expression Omnibus public database (GSE121374). T-cell receptor $\alpha \beta$ chain repertoires were aligned and assembled using software MiXCR (version 2.1.12). ${ }^{45}$ The TCRa $\beta$ clonotypes were exported respectively by parameter '-chains' in exportClones command of MiXCR. The exported clonotypes were visualized in the form of chord diagram using VDJtools software (version 1.1.10). ${ }^{72}$

\section{Statistics}

All quantitative analyses were based on at least three sample replicates. Data are presented as means \pm SD by GraphPad Prism. Independent-sample student $t$ test and One-way ANOVA were performed (SPSS). NS, not significant; ${ }^{*} P<0.05$; ${ }^{* *} P<0.01$; ${ }^{* * *} P<0.001$.

\section{ACKNOWLEDGEMENTS}

This work is supported by the Strategic Priority Research Program of Chinese Academy of Sciences (XDA16010601), the CAS Key Research Program of Frontier Sciences (QYZDB-SSW-SMC057), the Major Research and Development Project of Guangzhou Regenerative Medicine and Health Guangdong Laboratory (2018GZR110104006), the Chinese Ministry of Science and Technology (2015CB964401，2016YFA0100601，2017YFA0103401，2015CB964902, and 2015CB964901), the Health and Medical Care Collaborative Innovation Program of Guangzhou Scientific and Technology (201803040017), the Major Scientific and Technological Project of Guangdong Province (2014B020225005), the Science and Technology Planning Project of Guangdong Province (2017B030314056), the Program for Guangdong Introducing Innovative and Entrepreneurial Teams (2017ZT07S347), the National Natural Science Foundation of China (31471117, 81470281, 31600948, 31425012).

\section{AUTHOR CONTRIBUTIONS}

R.G. and F.H. conducted all the major experiments, data analysis and wrote the manuscript. Q.W., C.L., H.W., L.L., Y.Z., Z.B., M.Z., Y.L., X.L., C.X., T.W., P.Z., K.W., Y.D., Y.L., Y.G. and Y.G. participated in multiple experiments; Q.W. and Z.L. performed RNA-Seq and data analysis. C.L., H.W., Y.L., P.Z., Y.L., X.Z. and J.C. constructed vectors, prepared iPSC, designed and participated in gene editing. J.D.,Y.L. and Y.L. discussed the singlecell data; B.L. and J.W. discussed the data and wrote the manuscript; and J.W. designed the project and provided the final approval of the manuscript.

\section{ADDITIONAL INFORMATION}

Supplementary information accompanies this paper at https://doi.org/10.1038/ s41422-019-0251-7.

Competing interests: The authors declare no competing interests.

\section{REFERENCES}

1. Schmitt, T. M. \& Zuniga-Pflucker, J. C. Induction of T cell development from hematopoietic progenitor cells by delta-like-1 in vitro. Immunity 17, 749-756 (2002).

2. Mohtashami, M. et al. Direct comparison of Dll1- and DII4-mediated Notch activation levels shows differential lymphomyeloid lineage commitment outcomes. J. Immunol. 185, 867-876 (2010).

3. Montel-Hagen, A. et al. Organoid-Induced Differentiation of Conventional T Cells from Human Pluripotent Stem Cells. Cell Stem Cell 24, 376-389 e378 (2019).

4. Awong, G. et al. Human proT-cells generated in vitro facilitate hematopoietic stem cell-derived T-lymphopoiesis in vivo and restore thymic architecture. Blood 122, 4210-4219 (2013).

5. Shukla, S. et al. Progenitor T-cell differentiation from hematopoietic stem cells using Delta-like-4 and VCAM-1. Nat. Methods 14, 531-538 (2017).

6. Schmitt, T. M. et al. Induction of T cell development and establishment of T cell competence from embryonic stem cells differentiated in vitro. Nat. Immunol. 5, 410-417 (2004).

7. Sandler, V. M. et al. Reprogramming human endothelial cells to haematopoietic cells requires vascular induction. Nature 511, 312-318 (2014).

8. Sugimura, R. et al. Haematopoietic stem and progenitor cells from human pluripotent stem cells. Nature 545, 432-438 (2017).

9. Riddell, J. et al. Reprogramming committed murine blood cells to induced hematopoietic stem cells with defined factors. Cell 157, 549-564 (2014).

10. Lis, R. et al. Conversion of adult endothelium to immunocompetent haematopoietic stem cells. Nature 545, 439-445 (2017).

11. Stik, G. \& Graf, T. Hoxb5, a Trojan horse to generate T cells. Nat. Immunol. 19, 210-212 (2018).

12. Dzierzak, E. \& Bigas, A. Blood development: hematopoietic stem cell dependence and independence. Cell Stem Cell 22, 639-651 (2018).

13. Zhang, M. et al. Transcription factor Hoxb5 reprograms B cells into functional $T$ lymphocytes. Nat. Immunol. 19, 279-290 (2018).

14. Yoshimoto, M. et al. Autonomous murine T-cell progenitor production in the extra-embryonic yolk sac before HSC emergence. Blood 119, 5706-5714 (2012).

15. Luis, T. C. et al. Initial seeding of the embryonic thymus by immune-restricted lympho-myeloid progenitors. Nat. Immunol. 17, 1424-1435 (2016).

16. Tian, Y. et al. The first wave of T lymphopoiesis in zebrafish arises from aorta endothelium independent of hematopoietic stem cells. J. Exp. Med 214, 3347-3360 (2017).

17. Zeng, Y. et al. Single-cell RNA sequencing resolves spatiotemporal development of pre-thymic lymphoid progenitors and thymus organogenesis in human embryos. Immunity, https://doi.org/10.1016/j.immuni.2019.09.008. 
18. Yui, M. A. \& Rothenberg, E. V. Developmental gene networks: a triathlon on the course to T cell identity. Nat. Rev. Immunol. 14, 529-545 (2014).

19. Sroczynska, P., Lancrin, C., Kouskoff, V. \& Lacaud, G. The differential activities of Runx1 promoters define milestones during embryonic hematopoiesis. Blood 114, 5279-5289 (2009).

20. Chen, M. J., Yokomizo, T., Zeigler, B. M., Dzierzak, E. \& Speck, N. A. Runx1 is required for the endothelial to haematopoietic cell transition but not thereafter. Nature 457, 887-891 (2009).

21. North, T. E. et al. Runx1 expression marks long-term repopulating hematopoietic stem cells in the midgestation mouse embryo. Immunity 16, 661-672 (2002).

22. Wang, Q. et al. Disruption of the Cbfa2 gene causes necrosis and hemorrhaging in the central nervous system and blocks definitive hematopoiesis. Proc. Natl. Acad. Sci. USA 93, 3444-3449 (1996).

23. Okuda, T., van Deursen, J., Hiebert, S. W., Grosveld, G. \& Downing, J. R. AML1, the target of multiple chromosomal translocations in human leukemia, is essential for normal fetal liver hematopoiesis. Cell 84, 321-330 (1996).

24. Eilken, H. M., Nishikawa, S. \& Schroeder, T. Continuous single-cell imaging of blood generation from haemogenic endothelium. Nature 457, 896-900 (2009).

25. Pereira, C. F. et al. Induction of a hemogenic program in mouse fibroblasts. Cell Stem Cell 13, 205-218 (2013)

26. Pearson, S., Cuvertino, S., Fleury, M., Lacaud, G. \& Kouskoff, V. In vivo repopulating activity emerges at the onset of hematopoietic specification during embryonic stem cell differentiation. Stem Cell Rep. 4, 431-444 (2015).

27. So, C. W., Karsunky, H., Wong, P., Weissman, I. L. \& Cleary, M. L. Leukemic transformation of hematopoietic progenitors by MLL-GAS7 in the absence of Hoxa7 or Hoxa9. Blood 103, 3192-3199 (2004).

28. Ramos-Mejia, V. et al. HOXA9 promotes hematopoietic commitment of human embryonic stem cells. Blood 124, 3065-3075 (2014).

29. Magnusson, M. et al. HOXA10 is a critical regulator for hematopoietic stem cells and erythroid/megakaryocyte development. Blood 109, 3687-3696 (2007).

30. Komorowska, K. et al. Hepatic leukemia factor maintains quiescence of hematopoietic stem cells and protects the stem cell pool during regeneration. Cell Rep. 21, 3514-3523 (2017)

31. Davis, K. L. Ikaros: master of hematopoiesis, agent of leukemia. Ther. Adv. Hematol. 2, 359-368 (2011).

32. Nagel, S. et al. NKL homeobox gene activities in hematopoietic stem cells, T-cell development and T-cell leukemia. PLOS ONE 12, e0171164 (2017).

33. Oakley, K. et al. Setbp 1 promotes the self-renewal of murine myeloid progenitors via activation of Hoxa9 and Hoxa10. Blood 119, 6099-6108 (2012).

34. $\mathrm{Ng}, \mathrm{E}$. S. et al. Differentiation of human embryonic stem cells to $\mathrm{HOXA}(+)$ hemogenic vasculature that resembles the aorta-gonad-mesonephros. Nat. Biotechnol. 34, 1168-1179 (2016).

35. Zhou, F. et al. Tracing haematopoietic stem cell formation at single-cell resolution. Nature 533, 487-492 (2016).

36. Inlay, M. A. et al. Identification of multipotent progenitors that emerge prior to hematopoietic stem cells in embryonic development. Stem Cell Rep. 2, 457-472 (2014).

37. He, X., Park, K. \& Kappes, D. J. The role of ThPOK in control of CD4/CD8 lineage commitment. Annu Rev. Immunol. 28, 295-320 (2010).

38. Germar, K. et al. T-cell factor 1 is a gatekeeper for T-cell specification in response to Notch signaling. Proc. Natl. Acad. Sci. USA 108, 20060-20065 (2011).

39. Aliahmad, P., Seksenyan, A. \& Kaye, J. The many roles of TOX in the immune system. Curr. Opin. Immunol. 24, 173-177 (2012).

40. Palacios, E. H. \& Weiss, A. Function of the Src-family kinases, Lck and Fyn, in T-cell development and activation. Oncogene 23, 7990-8000 (2004).

41. Taghon, T., Yui, M. A. \& Rothenberg, E. V. Mast cell lineage diversion of T lineage precursors by the essential T cell transcription factor GATA-3. Nat. Immunol. 8, 845-855 (2007)

42. Liu, P., Li, P. \& Burke, S. Critical roles of Bcl11b in T-cell development and maintenance of T-cell identity. Immunol. Rev. 238, 138-149 (2010).

43. Hahm, K. et al. Helios, a T cell-restricted Ikaros family member that quantitatively associates with Ikaros at centromeric heterochromatin. Genes Dev. 12, 782-796 (1998)

44. Halim, T. Y. et al. Retinoic-acid-receptor-related orphan nuclear receptor alpha is required for natural helper cell development and allergic inflammation. Immunity 37, 463-474 (2012)

45. Bolotin, D. A. et al. MiXCR: software for comprehensive adaptive immunity profiling. Nat. Methods 12, 380-381 (2015).

46. Garrett-Sinha, L. A. Review of Ets1 structure, function, and roles in immunity. Cell Mol. Life Sci. 70, 3375-3390 (2013).

47. Zohren, F. et al. The transcription factor Lyl-1 regulates lymphoid specification and the maintenance of early T lineage progenitors. Nat. Immunol. 13, 761-769 (2012).

48. Hock, H. et al. Tel/Etv6 is an essential and selective regulator of adult hematopoietic stem cell survival. Genes Dev. 18, 2336-2341 (2004).
49. Greig, K. T., Carotta, S. \& Nutt, S. L. Critical roles for c-Myb in hematopoietic progenitor cells. Semin Immunol. 20, 247-256 (2008).

50. Wilkinson, A. C. et al. Single-cell analyses of regulatory network perturbations using enhancer-targeting TALEs suggest novel roles for PU.1 during haematopoietic specification. Development 141, 4018-4030 (2014).

51. Pang, S. H. M. et al. PU.1 Is Required for the Developmental Progression of Multipotent Progenitors to Common Lymphoid Progenitors. Front Immunol. 9, 1264 (2018).

52. Sugimura, R. The significance and application of vascular niche in the development and maintenance of hematopoietic stem cells. Int J. Hematol. 107, 642-645 (2018).

53. Azcoitia, V., Aracil, M., Martinez, A. C. \& Torres, M. The homeodomain protein Meis1 is essential for definitive hematopoiesis and vascular patterning in the mouse embryo. Dev. Biol. 280, 307-320 (2005).

54. Nam, C. H. \& Rabbitts, T. H. The role of LMO2 in development and in T cell leukemia after chromosomal translocation or retroviral insertion. Mol. Ther. 13 15-25 (2006).

55. Yu, Y. et al. Bcl11a is essential for lymphoid development and negatively regulates p53. J. Exp. Med. 209, 2467-2483 (2012)

56. Boutboul, D. et al. Dominant-negative IKZF1 mutations cause a T, B, and myeloid cell combined immunodeficiency. J. Clin. Invest. 128, 3071-3087 (2018).

57. Weng, A. P. et al. c-Myc is an important direct target of Notch1 in T-cell acute lymphoblastic leukemia/lymphoma. Genes Dev. 20, 2096-2109 (2006).

58. Del Real, M. M. \& Rothenberg, E. V. Architecture of a lymphomyeloid developmental switch controlled by PU.1, Notch and Gata3. Development 140, 1207-1219 (2013).

59. Uehara, S. et al. Premature expression of chemokine receptor CCR9 impairs T cell development. J. Immunol. 176, 75-84 (2006).

60. Zlotoff, D. A. et al. CCR7 and CCR9 together recruit hematopoietic progenitors to the adult thymus. Blood 115, 1897-1905 (2010).

61. Tussiwand, R. et al. The preTCR-dependent DN3 to DP transition requires Notch signaling, is improved by CXCL12 signaling and is inhibited by IL-7 signaling. Eur. J. Immunol. 41, 3371-3380 (2011).

62. Janas, M. L. et al. Thymic development beyond beta-selection requires phosphatidylinositol 3-kinase activation by CXCR4. J. Exp. Med. 207, 247-261 (2010).

63. Lawrence, $\mathrm{H}$. J. et al. Mice bearing a targeted interruption of the homeobox gene HOXA9 have defects in myeloid, erythroid, and lymphoid hematopoiesis. Blood 89, 1922-1930 (1997).

64. Gwin, K. A., Shapiro, M. B., Dolence, J. J., Huang, Z. L. \& Medina, K. L. Hoxa9 and Flt3 signaling synergistically regulate an early checkpoint in lymphopoiesis. $J$. Immunol. 191, 745-754 (2013).

65. Desbaillets, I., Ziegler, U., Groscurth, P. \& Gassmann, M. Embryoid bodies: an in vitro model of mouse embryogenesis. Exp. Physiol. 85, 645-651 (2000).

66. Chen, J. et al. Rational optimization of reprogramming culture conditions for the generation of induced pluripotent stem cells with ultra-high efficiency and fast kinetics. Cell Res 21, 884-894 (2011).

67. Lan, P., Tonomura, N., Shimizu, A., Wang, S. \& Yang, Y. G. Reconstitution of a functional human immune system in immunodeficient mice through combined human fetal thymus/liver and CD34+ cell transplantation. Blood 108, 487-492 (2006).

68. Jiang, $X$. et al. Skin infection generates non-migratory memory CD8+ T(RM) cells providing global skin immunity. Nature 483, 227-231 (2012).

69. Kim, D., Langmead, B. \& Salzberg, S. L. HISAT: a fast spliced aligner with low memory requirements. Nat. Methods 12, 357-360 (2015).

70. Pertea, $M$. et al. StringTie enables improved reconstruction of a transcriptome from RNA-seq reads. Nat. Biotechnol. 33, 290-295 (2015).

71. Pertea, M., Kim, D., Pertea, G. M., Leek, J. T. \& Salzberg, S. L. Transcript-leve expression analysis of RNA-seq experiments with HISAT, StringTie and Ballgown. Nat. Protoc. 11, 1650-1667 (2016).

72. Shugay, M. et al. VDJtools: unifying Post-analysis of T Cell Receptor Repertoires. PLoS Comput Biol. 11, e1004503 (2015).

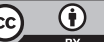

Open Access This article is licensed under a Creative Commons Attribution 4.0 International License, which permits use, sharing, adaptation, distribution and reproduction in any medium or format, as long as you give appropriate credit to the original author(s) and the source, provide a link to the Creative Commons license, and indicate if changes were made. The images or other third party material in this article are included in the article's Creative Commons license, unless indicated otherwise in a credit line to the material. If material is not included in the article's Creative Commons license and your intended use is not permitted by statutory regulation or exceeds the permitted use, you will need to obtain permission directly from the copyright holder. To view a copy of this license, visit http://creativecommons. org/licenses/by/4.0/.

(c) The Author(s) 2019 